\title{
The 2008 outburst in the young stellar system Z CMa
}

\section{Multi-epoch high-angular resolution images and spectra of the components in near-infrared}

\author{
M. Bonnefoy ${ }^{1,2}$, G. Chauvin ${ }^{1,2}$, C. Dougados ${ }^{1,2}$, Á. Kóspál ${ }^{3}$, M. Benisty ${ }^{1,2}$, G. Duchêne ${ }^{1,2,4}$, J. Bouvier ${ }^{1,2}$, \\ P. J. V. Garcia ${ }^{5}$, E. Whelan ${ }^{6}$, S. Antoniucci ${ }^{7}$, and L. Podio ${ }^{8}$ \\ ${ }^{1}$ Univ. Grenoble Alpes, IPAG, 38000 Grenoble, France \\ e-mail: mickael.bonnefoy@univ-grenoble-alpes.fr \\ 2 CNRS, IPAG, 38000 Grenoble, France \\ 3 Konkoly Observatory, Research Centre for Astronomy and Earth Sciences, Hungarian Academy of Sciences, PO Box 67, \\ 1525 Budapest, Hungary \\ 4 Astronomy Department, UC Berkeley, 501 Campbell Hall, Berkeley CA 94720-3411, USA \\ 5 Universidade do Porto, Faculdade de Engenharia, Rua Dr. Roberto Frias, s/n, 4200-465 Porto, Portugal \\ 6 Department of Experimental Physics, National University of Ireland Maynooth, Maynooth, Co. Kildare, Ireland \\ 7 INAF-Osservatorio Astronomico di Roma, via Frascati 33, 00078 Monte Porzio Catone, Italy \\ 8 INAF-Osservatorio Astrofisico di Arcetri, L.go E. Fermi 5, 50125 Firenze, Italy
}

Received 11 April 2016 / Accepted 12 June 2016

\begin{abstract}
Context. Z CMa is a complex pre-main sequence binary with a current separation of 110 mas, known to consist of an FU Orionis star (SE component) and an embedded Herbig Be star (NW component). Although it represents a well-studied and characterized system, the origin of photometric variabilities, the component properties, and the physical configuration of the system remain mostly unknown.

Aims. Immediately when the late-2008 outburst of Z CMa was announced to the community, we initiated a high angular resolution imaging campaign aimed at characterizing the outburst state of both components of the system in the near-infrared.

Methods. We used the VLT/NACO and the Keck/NIRC2 near-infrared adaptive optics instrument to monitor the astrometric position and the near-infrared photometry of the Z CMa components during the outburst phase and one year after. The VLT/SINFONI and Keck/OSIRIS integral field spectroscrographs were in addition used to characterize for the first time the resolved spectral properties of the FU Orionis and the Herbig Be component during and after the outburst.

Results. We confirm that the NW star dominates the system flux in the 1.1-3.8 $\mu \mathrm{m}$ range and is responsible for the photometric outburst. We extract the first medium-resolution $(R \sim 2000-4000)$ near-infrared (1.1-2.4 $\mu \mathrm{m})$ spectra of the individual components. The SE component has a spectrum typical of FU Orionis objects. The NW component spectrum is characteristic of embedded outbursting protostars and EX Or objects. It displays numerous emission lines whose intensity correlates with the system activity. In particular, we find a correlation between the Bry equivalent width and the system brightness. The bluing of the continuum of the NW component along with the absolute flux and color-variation of the system during the outburst suggests that the outburst was caused by a complex interplay between a variation of the extinction in the line of sight of the NW component on one hand, and the emission of shocked regions close to the NW component on the other. We confirm the recently reported wiggling of the SE component jet from [Fe II] line emission. We find a point-like structure associated with a peak emission at $2.098 \mu \mathrm{m}$ coincidental with the clump or arm seen in broadband polarization differential imaging as well as additional diffuse emission along a $\mathrm{PA}=214^{\circ}$. The origin of these two structures is unclear and deserves further investigation.
\end{abstract}

Key words. techniques: high angular resolution - binaries: general - stars: individual: Z CMa - stars: pre-main sequence

\section{Introduction}

$\mathrm{Z}$ Canis Majoris (Z CMa) is a complex pre-main sequence binary member of the CMa OB1 association (age < 1 Myr; Herbst et al. 1978), with an estimated distance ranging from $950 \mathrm{pc}$ to 1150 pc (Clariá 1974; Humphreys 1978; Kaltcheva \& Hilditch 2000). The system is known for its recurring EX Orionis (EX Or)-like outbursts with recent events recorded in 1987, 2000, 2004, 2008, 2011, 2015, and 2016.

Z CMa was classified by Hartmann et al. (1989) as a FU Orionis variable based on its broad, doubled optical line absorptions, optical spectral type of F-G, and CO first overtone. The authors were able to reproduce the UV-optical spectrum by a model of an optically thick accretion disk surrounding an 1-3 $M_{\odot}$ star with an accretion rate of $10^{-3} M_{\odot} \mathrm{yr}^{-1}$. The model failed to explain the near-infrared (NIR) part of the Z CMa spectrum, however, as well as a strong increase in brightness in 1987. During the 1987 outburst, the optical spectrum was characterized by a featureless and bluer continuum, a rise of Balmer lines, and emission lines of Fe II, Cr II, and Ti II (Hessman et al. 1991), which is unconsistent with typical FU Orionis activity.

The problem was partly solved by Koresko et al. (1991) who discovered that Z CMa consists of a 110 mas binary. The SW component of the pair is the FU Orionis star that dominates the optical flux. The NW component dominates the NIR $(>2 \mu \mathrm{m})$ to sub-mm spectrum, and the total luminosity. 


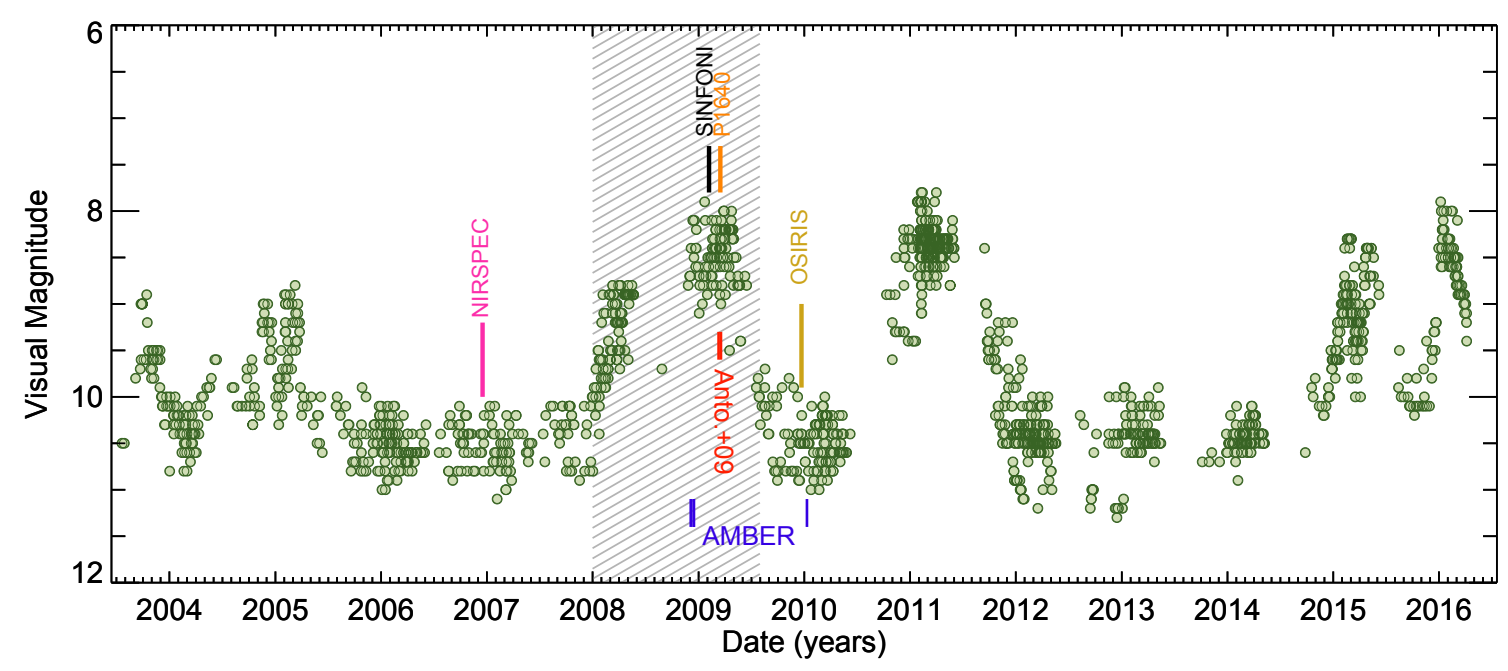

Fig. 1. Light curve of Z CMa inferred from AAVSO observations. The spectroscopic observations of the HBe and FUOR are reported in the figure. The dashed zone corresponds to the 2008 outburst of the system.

Follow-up observations (Whitney et al. 1993; Garcia et al. 1999; van den Ancker et al. 2004, hereafter VDA04) permitted the conclusion that this component is an Herbig Be star (hereafter $\mathrm{HBe})$. VDA04 obtained a NIR $(1.9-4.1 \mu \mathrm{m})$ spectrum while the system was returning to its quiescent state. This spectrum shows $\operatorname{Br} \alpha, \operatorname{Br} \gamma$, and Pf $\gamma$ in emission and the CO band-head in absorption around $2.3 \mu \mathrm{m}$. The authors suggested that the emission lines are formed into an accreting circumstellar disk surrounding the $\mathrm{HBe}$, and also in an extended envelope above and below the disk plane. The emission line analysis led VDA04 to infer a B0IIIe spectral type. Luminosity and effective temperature estimates enable the authors to derive a mass of $16-38 M_{\odot}$ for the $\mathrm{HBe}$ using various evolutionary model predictions for ages of $3 \times 10^{5}$ yr and $4 \times 10^{4}$ yr respectively.

At large scales, this system reveals a rich outflow activity. A collimated optical outflow $\left(3.9 \mathrm{pc} ; \mathrm{PA}=240^{\circ}\right)$ and a bowshock-shaped feature at $60^{\prime \prime}$ have been reported by Poetzel et al. (1989). Multiple outflow component profiles traced by optical forbidden lines are present close to the source. Whelan et al. (2010) confirmed the existence of a jet driven by the $\mathrm{HBe}$ star (paper II), which is unambiguously the driving source of the Z CMa parsec-scale outflow. The HBe star jet is seen oscillating around a given position angle (jet wiggling), probably due to a closer companion to the HBe star. A twin jet driven by the FUOR component was also clearly identified. Millan-Gabet et al. (2001) also reported a cavity-like structure in adaptive optics $J$-band imaging, extending to the SW of the system, which they interpreted as light scattered off the wall of a jet-blown cavity aligned with the Z CMa large-scale outflows.

Alonso-Albi et al. (2009) modeled the spectral energy distribution of the system by contributions from free-free emission, a disk of size $180_{-140}^{+250}$ au tilted by $30_{-20}^{+40}$ degrees, and an infalling envelope (spherical or toroidal) surrounding each star that possibly extends from 2000 to 5000 au and might be carved by the outflows. The $\mathrm{HBe}$ itself is embedded in a dust cocoon (Whitney et al. 1993). A sketch of the system can be found in Canovas et al. (2012). The dust distribution at the 500 au scale around Z CMa was investigated using NIR imaging polarimetry (Canovas et al. 2015; Liu et al. 2016). It reveals an extended filamentary structure (up to $2^{\prime \prime}$ ) observed by Millan-Gabet \& Monnier (2002), whose origin is unclear, as well as a polarized clump closer to the stars $\left(0.3-0.5^{\prime \prime}\right)$.
At the Astronomical Unit scale, the FUOR is strongly resolved by the Keck-I interferometer to a level that is difficult to explain with thermal emission of the accretion disk alone (Millan-Gabet et al. 2006). Hence its close environment appears to be more complex than expected. The HBe component has also been observed with Keck-I (Monnier et al. 2005) and with AMBER on the VLTI (Li Causi et al. 2008; Benisty et al. 2010). Based on the Keck observations, the authors have modelled the NIR emission as coming from a uniform ring. Nonetheless, they have finally concluded that the ring model was doubtful for this star, considering the large uncertainty of its spectral type. Morover, the amount of infrared emission coming from this source alone at a given epoch is also poorly known, which adds to the difficulty of interpreting the interferometric measurements.

From January 2008 to October 2009, the system began to experience an optical outburst, the largest reported in the past 90 years of available observations (Grankin \& Artemenko 2009). This outburst triggered additional observations of the system. Hinkley et al. (2013) presented adaptive optics $J H K L$ band photometry of the individual components. They concluded that the embedded $\mathrm{HBe}$ component is solely responsible for the outburst. Benisty et al. (2010) obtained spectrally resolved interferometric observations of the $\mathrm{HBe}$ to study the hot gas emitting across the $\mathrm{Br} \gamma$ emission line (paper I). They found that the line profile, the astrometric signal, and the visibilities are consistent with the emission of a bipolar wind that may be partly seen through a disk hole inside the dust sublimation radius at the au scale. Their multi-epoch observations led them to suggest that the outburst is related to a period of strong mass-loss and not to a change in the extinction along the line of sight. The spectrophotometric, spectropolarimetric, and polarimetric imaging observations of the system (Szeifert et al. 2010; Canovas et al. 2012) suggest in contrast that the outburst was caused by changes in extinction of the dust cocoon surrounding the HBe star.

We present the results of a complementary high angular resolution imaging campaign to resolve and characterize the properties of each component of Z CMa in the NIR during and after its 2008 outburst phase and to study its close environnement, in an attempt to better understand the origins of the bursts (extinction, or accretion and ejection scenarios). Our new observations were made from January to March 2009, that is, about one year after the start of the two-year outburst. We obtained an additional observation when the system returned to quiescence (see Fig. 1). 
Table 1. Observing log. Sr- $2.2 \mu \mathrm{m}$ corresponds to the Strehl ratio measured at $2.2 \mu \mathrm{m}$.

\begin{tabular}{|c|c|c|c|c|c|c|c|c|}
\hline UT Date & Name & Instr. & Filter (Grism) & Camera & Exp. time & Sr-2.2 $\mu \mathrm{m}$ & Airmass & Comment \\
\hline 2009-01-31 & $\begin{array}{l}\text { Z CMa } \\
\text { HD54335 }\end{array}$ & $\begin{array}{l}\text { NACO } \\
\text { NACO }\end{array}$ & $\begin{array}{l}J, H, K_{\mathrm{s}}, L^{\prime} \\
J, H, K_{\mathrm{s}}, L^{\prime}\end{array}$ & $\begin{array}{l}\text { S13, L27 } \\
\text { S13, L27 }\end{array}$ & $\begin{array}{l}90 \mathrm{~s} \\
90 \mathrm{~s}\end{array}$ & $\begin{array}{l}(32 \%) \\
22 \%\end{array}$ & $\begin{array}{l}1.19 \\
1.25\end{array}$ & psf-ref \\
\hline 2009-02-06 & $\begin{array}{l}\text { Z CMa } \\
\text { Z CMa } \\
\text { Z CMa }\end{array}$ & $\begin{array}{l}\text { SINFONI } \\
\text { SINFONI } \\
\text { SINFONI }\end{array}$ & $\begin{array}{l}J \\
H \\
K\end{array}$ & $\begin{array}{l}0.025 \\
0.025 \\
0.025\end{array}$ & $\begin{array}{l}240 \mathrm{~s} \\
240 \mathrm{~s} \\
240 \mathrm{~s}\end{array}$ & $\begin{array}{l}\text { n.a. } \\
\text { n.a. } \\
\text { n.a. }\end{array}$ & $\begin{array}{l}1.05 \\
1.09 \\
1.22\end{array}$ & \\
\hline $\begin{array}{l}2009-01-28 \\
2009-02-22 \\
2009-02-07\end{array}$ & $\begin{array}{l}\text { HIP072241 } \\
\text { HIP054970 } \\
\text { HIP071218 }\end{array}$ & $\begin{array}{l}\text { SINFONI } \\
\text { SINFONI } \\
\text { SINFONI }\end{array}$ & $\begin{array}{l}J \\
H \\
K\end{array}$ & $\begin{array}{l}0.25 \\
0.25 \\
0.25\end{array}$ & $\begin{array}{l}10 \mathrm{~s} \\
12 \mathrm{~s} \\
10 \mathrm{~s}\end{array}$ & $\begin{array}{l}\text { n.a. } \\
\text { n.a. } \\
\text { n.a. }\end{array}$ & $\begin{array}{l}1.06 \\
1.09 \\
1.22\end{array}$ & $\begin{array}{l}\text { telluric } \\
\text { telluric } \\
\text { telluric }\end{array}$ \\
\hline $2009-02-26$ & $\begin{array}{l}\text { Z CMa } \\
\text { HD54335 }\end{array}$ & $\begin{array}{l}\text { NACO } \\
\text { NACO }\end{array}$ & $\begin{array}{l}J, H, K_{\mathrm{s}}, L^{\prime} \\
J, H, K_{\mathrm{s}}, L^{\prime}\end{array}$ & $\begin{array}{l}\text { S13, L27 } \\
\text { S13, L27 }\end{array}$ & $\begin{array}{l}90 \mathrm{~s} \\
90 \mathrm{~s}\end{array}$ & $\begin{array}{l}(42 \%) \\
41 \%\end{array}$ & $\begin{array}{l}1.06 \\
1.11\end{array}$ & psf-ref \\
\hline 2009-03-11 & $\begin{array}{l}\text { Z CMa } \\
\text { HD54335 }\end{array}$ & $\begin{array}{l}\text { NACO } \\
\text { NACO }\end{array}$ & $\begin{array}{l}J, H, K_{\mathrm{s}}, L^{\prime} \\
J, H, K_{\mathrm{s}}, L^{\prime}\end{array}$ & $\begin{array}{l}\text { S13, L27 } \\
\text { S13, L27 }\end{array}$ & $\begin{array}{l}90 \mathrm{~s} \\
90 \mathrm{~s}\end{array}$ & $\begin{array}{l}(41 \%) \\
34 \%\end{array}$ & $\begin{array}{l}1.02 \\
1.04\end{array}$ & psf-ref \\
\hline 2009-12-07 & $\begin{array}{l}\text { Z CMa } \\
\text { HIP33998 }\end{array}$ & $\begin{array}{l}\text { NIRC2 } \\
\text { NIRC2 }\end{array}$ & $\begin{array}{l}J_{\text {cont }}, H_{\text {cont }}, K_{\text {cont }} \\
J_{\text {cont }}, H_{\text {cont }}, K_{\text {cont }}\end{array}$ & $\begin{array}{l}9.96 \mathrm{mas} / \mathrm{pix} \\
9.96 \mathrm{mas} / \mathrm{pix}\end{array}$ & $\begin{array}{l}8.6 \mathrm{~s} \\
8.6 \mathrm{~s}\end{array}$ & - & $\begin{array}{l}1.29 \\
1.51\end{array}$ & psf-ref \\
\hline
\end{tabular}

Notes. Seeing evaluated at $0.55 \mu \mathrm{m}$.

We extracted the first medium-resolution $(R \sim 2000-4000)$ spectra from 1.1 to $2.5 \mu \mathrm{m}$ of each components of the system. We present additional $J H K L$ band photometric and astrometric data of the binary. We report in Sect. 2 our observations and detail the associated data reduction and analysis in Sect. 3. We analyze the evolution of the NIR photometric and spectroscopic properties of the components in Sects. 4 and 5, respectively. We discuss these results in the context of other variable objects, and of past outbursts of the system in Sect. 6. We summarize our results in Sect. 7.

\section{Observations}

\subsection{VLT/NACO}

The Z CMa 2008 outburst was monitored with the NACO highcontrast adaptive optics (AO) imager of the VLT-UT4. The NAOS AO system (Rousset et al. 2003) is equipped with a tip-tilt mirror, a 185 piezo actuator deformable mirror and two wavefront sensors (visible and IR). Attached to NAOS, CONICA (Lenzen et al. 2003) is the NIR (1-5 $\mu$ m domain) imaging camera equipped with a $1024 \times 1024$ pixel Aladdin InSb array. Observations were obtained at three different epochs between January 2009 and March 2009 (outburst phase). During the different observing campaigns, the atmospheric conditions were sufficiently stable to close the AO loop and resolve both components (see Fig. 2, left panel). The fainter FU Orionis component in $K$-band is clearly visible on both Keck and VLT images. Both images were normalized in flux to show the flux variation that is discernable between January and December 2009 in $K$ band. The relative position of the FUOR with respect to that of the HBe component could be monitored well. The typical observing sequence included a set of five jittered images obtained using the $J, H$, and $K_{\mathrm{s}}$ bands with the $\mathrm{S} 13$ camera CONICA (mean plate scale of 13.25 mas/pixel) and using the $L^{\prime}$ filter with L27 (mean plate scale of $27.10 \mathrm{mas} / \mathrm{pixel}$ ), leading to a total exposure time of $\sim 5 \mathrm{~min}$ on source. The corresponding setups are reported in Table 1.

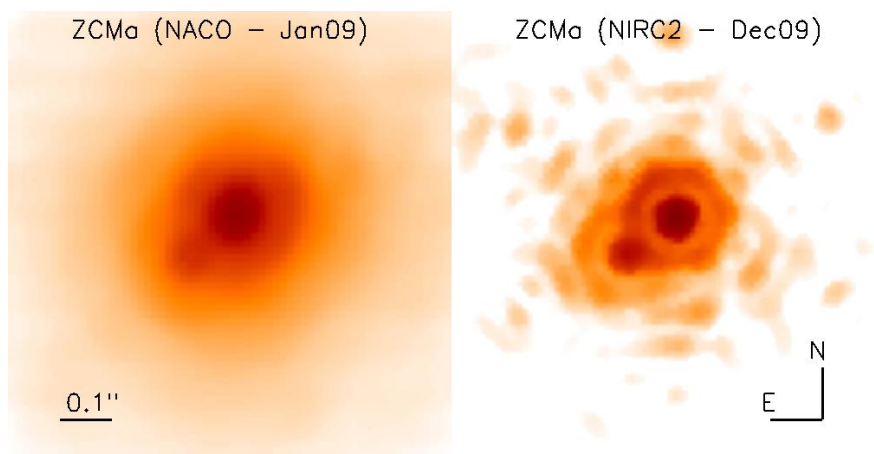

Fig. 2. Left: VLT/NACO $K_{\mathrm{s}}$-band image of Z CMa on Januray 31, 2009. Right: Keck/NIRC2 $K_{\text {cont }}$ image on December 7, 2009.

\section{2. $\mathrm{Keck} / \mathrm{NIRC2}$}

On December 17, 2009 (transient phase), imaging observations were also obtained using the $\mathrm{AO}$ system on the $10 \mathrm{~m}$ Keck II telescope (van Dam et al. 2004). We obtained direct images from 1.0 to $2.5 \mu \mathrm{m}$ that clearly resolved the two components of the system with the facility AO-dedicated NIR camera NIRC2, a $1024 \times 1024$ detector for which we used the $9.963 \pm 0.005$ mas pixel $^{-1}$ scale and whose absolute orientation on the sky is $0.13 \pm 0.02^{\circ}$ (Ghez et al. 2008). We used the narrowband filters $J_{\text {cont }}, H_{\text {cont }}$, and $K_{\text {cont }}$ centered on $1.2132,1.5804$, and $2.2706 \mu \mathrm{m}$ to avoid the saturation of the star. The corresponding setups are also reported in Table 1. Both components were easily resolved to determine the relative flux and position (Fig. 2, right panel).

\subsection{VLT/SINFONI}

We used the SINFONI instrument (Spectrograph for INfrared Field Observations, see Eisenhauer et al. 2003a; Bonnet et al. 2004), located at the Cassegrain focus of the VLT UT4 Yepun to observe Z CMa during the outburst phase on February 6, 2009. The instrument provides AO-assisted integral field spectroscopy. It uses a modified version of the Multi-Applications Curvature Adaptive Optics system (MACAO, Bonnet et al. 2003) designed to feed the SPectrograph for Infrared Faint Field Imaging 
Table 2. NaCo and Keck relative astrometry and photometry of FUOR relative to the HBe star in $J H K_{\mathrm{s}}$.

\begin{tabular}{lllllllll}
\hline \hline UT Date & $\begin{array}{l}\Delta \\
(\mathrm{mas})\end{array}$ & $\begin{array}{l}\text { PA } \\
(\mathrm{deg})\end{array}$ & $\begin{array}{l}\text { Platescale } \\
(\mathrm{mas})\end{array}$ & $\begin{array}{l}\text { True north } \\
(\mathrm{deg})\end{array}$ & $\begin{array}{l}\Delta J \\
(\mathrm{mag})\end{array}$ & $\begin{array}{l}\Delta H \\
(\mathrm{mag})\end{array}$ & $\begin{array}{l}\Delta K_{\mathrm{s}} \\
(\mathrm{mag})\end{array}$ & $\begin{array}{l}\Delta L^{\prime} \\
(\mathrm{mag})\end{array}$ \\
\hline $09-01-31$ & $111 \pm 3$ & $132.7 \pm 1.0$ & & & $1.19 \pm 0.13$ & $1.94 \pm 0.10$ & $2.42 \pm 0.11$ & $2.9 \pm 0.3$ \\
$09-02-26$ & $111 \pm 3$ & $132.4 \pm 1.0$ & $13.25 \pm 0.04$ & $0.10 \pm 0.15$ & $1.16 \pm 0.04$ & $1.46 \pm 0.04$ & $1.97 \pm 0.05$ & \\
$09-03-11$ & $112 \pm 3$ & $132.1 \pm 1.0$ & & & $0.82 \pm 05$ & $1.41 \pm 0.05$ & $2.01 \pm 0.04$ & \\
$09-12-07$ & $111 \pm 3$ & $133.3 \pm 1.0$ & $9.963 \pm 0.005$ & $0.13 \pm 0.02$ & $-0.47 \pm 0.20$ & $0.64 \pm 0.05$ & $1.51 \pm 0.05$ & \\
\hline
\end{tabular}

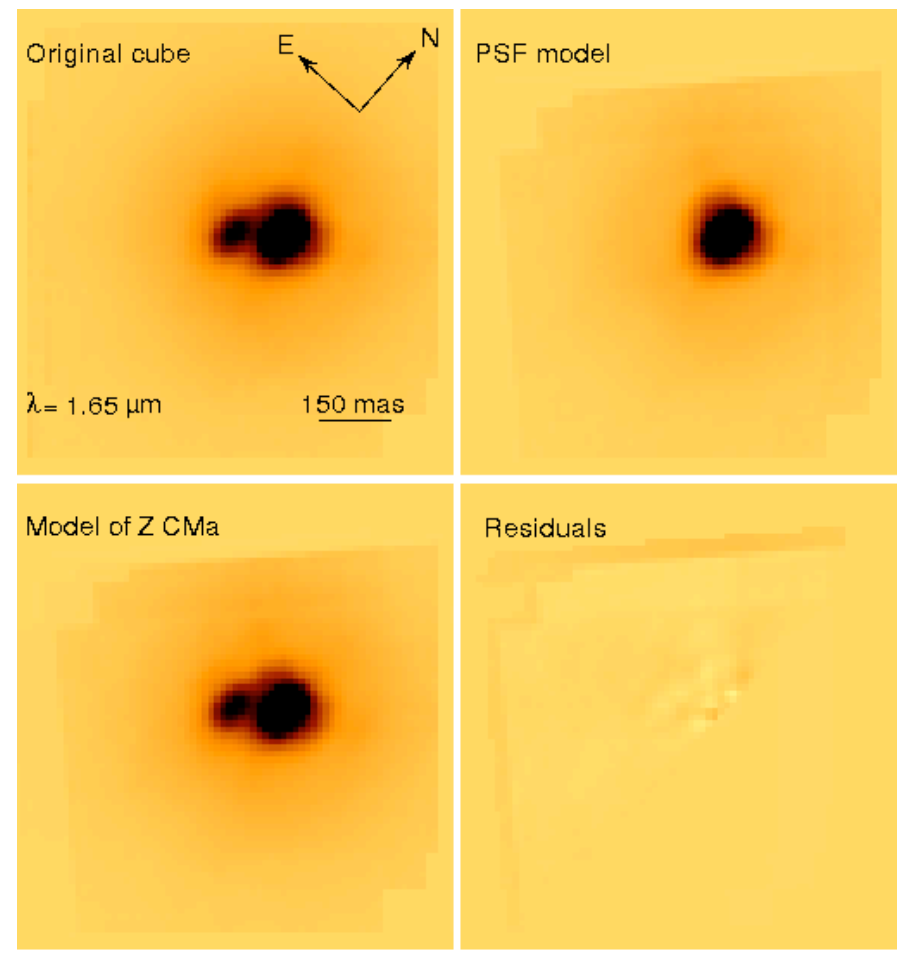

Fig. 3. Illustration of the spectral deblending process at $1.65 \mu \mathrm{m}$. Upper left: the initial Z CMa datacube where the two components are resolved. Upper right: during the observation, the adaptive-optics corrected PSF showed a strong astigmatism. To achieve a proper extraction of the spectra of the Z CMa components, we duplicated the HBe star profile to create a PSF model. Lower left: the position and the flux of the individual sources were then retrieved using a modified version of the CLEAN algorithm. Lower right: the extraction error is estimated from the residuals.

(SPIFFI, Eisenhauer et al. 2003b). SPIFFI pre-slit optics where chosen to provide a spatial pixel scale of $12.5 \times 25$ mas per pixel. Three different gratings were used to cover the $J(1.1-1.4 \mu \mathrm{m})$, $H(1.45-1.85 \mu \mathrm{m})$, and $K$ band $(1.95-2.45 \mu \mathrm{m})$ at medium resolving powers (2000, 3000, and 4000 respectively).

The instrument was rotated to orient the binary horizontally in the field of view (FoV) (see Fig. 2). Sky exposures were recorded following a $\mathrm{ABBA}$ pattern. Additional offsets on the object were chosen to increase the FOV to $850 \times 860$ mas in the $J$ band, $850 \times 900$ mas in the $H$ band, and $950 \times 900$ mas in the $K$ band. This also permitted us to artificially double the vertical spatial sampling. The source was bright enough at optical wavelengths to be used as guide probe for the wavefront sensing. HIPPARCOS standard stars were also acquired soon after $\mathrm{Z} \mathrm{CMa}$ to correct the spectra for telluric features (see Table 1).

\section{Data analysis}

\subsection{High-contrast imaging}

After cosmetic reductions (bad pixels, dark subtraction, flat fielding) using eclipse (Devillard 1997), we applied the deconvolution algorithm of Veran \& Rigaut (1998) to obtain the position of the HBe component relative to the FUOR at each epoch for both $\mathrm{NaCo}$ and Keck data. The star HD54335 ( $V=7.4$, $K=3.6)$ and HIP33998 ( $V=7.99, K=7.895)$ of similar brightness were observed consecutively to serve as point spread function (PSF) estimate for the $\mathrm{NaCo}$ and Keck data, respectively. The results are reported in Table 2 . The total system brightness was calibrated using the zero points provided by ESO for each observing night, or using the PSF standard HIP33998 observed with NIRC2.

\subsection{Integral field spectroscopy}

SINFONI data were reduced with the ESO data reduction pipeline version 1.9.8 (Abuter et al. 2006). We used in addition custom routines to correct raw images from several electronic effects that affect the detector, as previously reported in Bonnefoy et al. (2014). The pipeline carried out cube reconstruction from corrected detector images. Hot and nonlinear pixels were tagged using dark and flat-field exposures. Arc lamp frames acquired during the days following the observations enabled us to calibrate the distortion, the wavelength scale, and the slitlet positions. Slitlet distances were measured with north-south scanning of the detector illuminated with an optical fiber. Objectsky frame pairs were subtracted, flat-fielded and corrected for bad pixels and distortions. In each band, four datacubes were finally reconstructed from clean science images and merged into a master cube. The quality of the reduction was checked using the ESO trending parameters. We obtained standard star datacubes in a similar way. However, their low signal to noise ratio $(\mathrm{S} / \mathrm{N})$ would have degraded the final Z CMa spectra, therefore we decided not to use it. We instead reduced datacubes of the telluric standards HIP072241 (B3V) in the $J$ band, HIP054970 (B5III) in the $H$ band, and of HIP HIP071218 (G2V) in the $K$ band observed at the airmass of $\mathrm{Z} \mathrm{CMa}$ on different nights and with the instrument pre-optics offering a 0.25 arcsecond/spaxel sampling. The standard star spectra and the Z CMA spectra were smoothed to the same resolution. This change of resolution was estimated from the arc lamp calibration files taken the same days as the observations. Those telluric standards were found to provide the best possible removal of the telluric lines.

Although Z CMa components are resolved in our final datacubes, the two sources contaminate each other. We therefore developed an improved version of the spectral extraction algorithm described in Bonnefoy et al. (2009) to retrieve deblended spectra of the HBe and FUOR stars in each band (see Fig. 2). 
M. Bonnefoy et al.: Multi-epoch images and spectra of the components of Z CMa in the near-infrared during the 2008 outburst

Table 3. NaCo and Keck photometry of HBe, FUOR, and of the system in $J H K_{\mathrm{s}}$.

\begin{tabular}{llllllllll}
\hline \hline UT Date & $J_{\mathrm{HBe}}$ & $H_{\mathrm{HBe}}$ & $K \mathrm{~s}_{\mathrm{HBe}}$ & $J_{\mathrm{FUOR}}$ & $H_{\mathrm{FUOR}}$ & $K \mathrm{~s}_{\mathrm{FUOR}}$ & $J_{\text {syst }}$ & $H_{\text {syst }}$ & $K \mathrm{~s}_{\text {syst }}$ \\
\hline $09-01-31$ & $6.22 \pm 0.08$ & $4.97 \pm 0.08$ & $3.75 \pm 0.09$ & $7.41 \pm 0.08$ & $6.91 \pm 0.08$ & $6.07 \pm 0.09$ & $5.91 \pm 0.07$ & $4.81 \pm 0.07$ & $3.63 \pm 0.07$ \\
$09-02-26$ & $6.13 \pm 0.08$ & $5.07 \pm 0.08$ & $3.98 \pm 0.09$ & $7.29 \pm 0.08$ & $6.53 \pm 0.08$ & $5.95 \pm 0.09$ & $5.81 \pm 0.07$ & $4.82 \pm 0.07$ & $3.82 \pm 0.07$ \\
$09-03-11$ & $6.55 \pm 0.09$ & $5.35 \pm 0.10$ & $3.95 \pm 0.07$ & $7.39 \pm 0.09$ & $6.76 \pm 0.10$ & $5.97 \pm 0.07$ & $6.14 \pm 0.07$ & $5.09 \pm 0.07$ & $3.79 \pm 0.07$ \\
$09-12-07$ & $7.6 \pm 0.2$ & $6.2 \pm 0.2$ & $3.6 \pm 0.2$ & $7.1 \pm 0.2$ & $6.9 \pm 0.2$ & $5.2 \pm 0.2$ & $6.59 \pm 0.2$ & $5.72 \pm 0.2$ & $3.39 \pm 0.2$ \\
\hline
\end{tabular}

Our algorithm, CLEAN 3D has now been applied successfully on different datasets (Bonnefoy et al. 2014; Bergfors et al. 2016). We provide a reference concise description in this paper. The positions of the two sources were initially fit with a Moffat function in each monochromatic cube slice. Their variation with wavelength caused by the atmospheric refraction was fit with a loworder polynomial. A modified version of the CLEAN algorithm (Dumas et al. 2001) was then applied on each slice to create a model of Z CMa while keeping the position of the binary fixed. The standard star datacubes were not reprentative of the PSF of $\mathrm{Z}$ CMa, which was affected by a strong astigmatism and could therefore not be used as input of the algorithm. We therefore chose to create a PSF template duplicating the Herbig profile following the observed direction of the PSF lengthening. The position of the sources were then refined, an improved PSF model was created and a second CLEAN step was performed on the original cube. The algorithm produced two final datacubes containing the model of the HBe and the FUOR components. The typical error introduced by the spectral extraction and computed from residual maps ranges from 0.5 to $5 \%$. Z CMa spectra were finally integrated inside circular appertures of 390 mas in the $J$ band and 1250 mas in the $H$ and $K$ band. Spectra were divided by the standard star spectra corrected for intrinsic features and from their black body. The standard star spectra are found to provide a good correction of telluric features in the $J$ band and a moderate correction in the $H$ and $K$ band. Spectra were finally scaled in flux by interpolating the NACO photometry of January 31, 2009 and February 26, 2009 on the date of the SINFONI observations. We estimate that the $\mathrm{S} / \mathrm{N}$ measured on linefree regions on the Herbig spectra is 205 in the $J$ band, 122 in the $H$ band, and 120 in the $K$ band.

We repeated this procedure on the $J b b(1.18-1.416 \mu \mathrm{m})$ and $H b b(1.473-1.803 \mu \mathrm{m}) \mathrm{Keck} / \mathrm{OSIRIS}$ datacubes obtained on December 22, 2009 (Whelan et al. 2010) when the system had returned to its quiescent state. We also reduced and analyzed an additional datacube obtained on the same night with the $K b b$ (1.965-2.381 $\mu \mathrm{m})$ setting. The cubes have $20 \times 20$ mas spaxels that sample the system separation well. The limited FoV of the cubes along the binary PA and residual artifacts limited the accuracy of the extraction to $\sim 10 \%$. We obtained the spectra of each component by integrating the flux of the sources over circular apertures $(R=5$ pixels $)$ in the extracted datacubes. The spectra were corrected for the atmospheric transmission using the spectra of A0V stars observed on the same night (HD 67213 for the $J b b$ and $K b b$ datacubes, HD 109615 for the $K b b$ datacubes). The standard star spectra were previously multiplied by a highresolution $(R=500000)$ spectrum of Vega smoothed at the resolution of OSIRIS $(R \sim 3800)$ and shifted to the radial velocity of the stars ${ }^{1}$. The standard star was located at the edge of the FoV of the $H$-band cube, which introduced strong differential flux losses

\footnotetext{
1 http://kurucz.harvard.edu/stars/VEGA/
}

that translate into an additional slope in the final spectra. We estimated and corrected the spectra for this slope considering that the FUOR spectral slope should be identical to the one of the SINFONI spectrum. The OSIRIS spectra have a lower $\mathrm{S} / \mathrm{N}$ than those of the SINFONI spectra $(S / N=31$ in the $J$ band, 82 in the $H$ band, and 70 in the $K$ band). Finally, we note that the $K$-band spectra are also slightly affected by an oscillating pattern (with a $0.08 \mu \mathrm{m}$ period) that might be related to numerical artifacts found on the spaxels close to the edges of the FoV.

\section{AO imaging of $Z \mathrm{CMa}$}

\subsection{Resolved photometry}

The photometry of the FUOR component relative the $\mathrm{HBe}$ at each epoch for both $\mathrm{NaCo}$ and Keck data is reported in Table 3 and compared in Fig. 4 with previous total and individual photometry. From the literature, the FUOR is brighter in the quiescent phase in $J$ and $H$ bands, whereas the HBe dominates in $K$ band. The system brightness also seems to decay significantly since 1986, and presumably since the last FUOR burst. During the 2008 outburst, our $\mathrm{NaCo}$ data show that the HBe component became much brighter in all NIR wavelengths than the FUOR, as shown by a flux ratio inversion in $J$ and $H$ bands (see Table 2). This confirms that the $\mathrm{HBe}$ component is at the origin of the 2008 outburst, in agreement with Hinkley et al. (2013). Our Keck observations, taken one year later, indicate that the system did not completely return to its quiescent phase in December 2009 as the $\mathrm{HBe}$ component remains brighter in $H$-band.

\subsection{Extended structures}

In our $2009 \mathrm{NaCo}$ observation in $J$ or $H$ bands, we did not redetect any extended structures because the Keck data have comparatively a higher $\mathrm{S} / \mathrm{N}$, as we did not intend to saturate our observations to derive the photometry of the two components. An extended structure at a projection angle (PA) compatible with that reported by Millan-Gabet \& Monnier (2002), Canovas et al. (2015), and Liu et al. (2016) is clearly seen in the $L^{\prime}$ band (annoted "A" in Fig. 5) after applying a radial profile subtraction. Its PA ranges from 160 to $250^{\circ}$ and extends up to $2160 \mathrm{au}$. Two other structures ("B" and " $\mathrm{C}$ " in Fig. 5) are marginally detected in this band at PA $\sim 0$ and $180^{\circ}$. They are not detected at shorter NIR wavelengths or in polarimetry (Canovas et al. 2015; Liu et al. 2016). Their orientation does not coincide with the shocked emission regions seen in the wide-field images of Z CMa (Poetzel et al. 1989; Bouy et al. 2011). They could be part of the cavity whose NW extension displays a complex structure (Poetzel et al. 1989) or be residuals from the stellar halo subtraction. 
A\&A 597, A91 (2017)
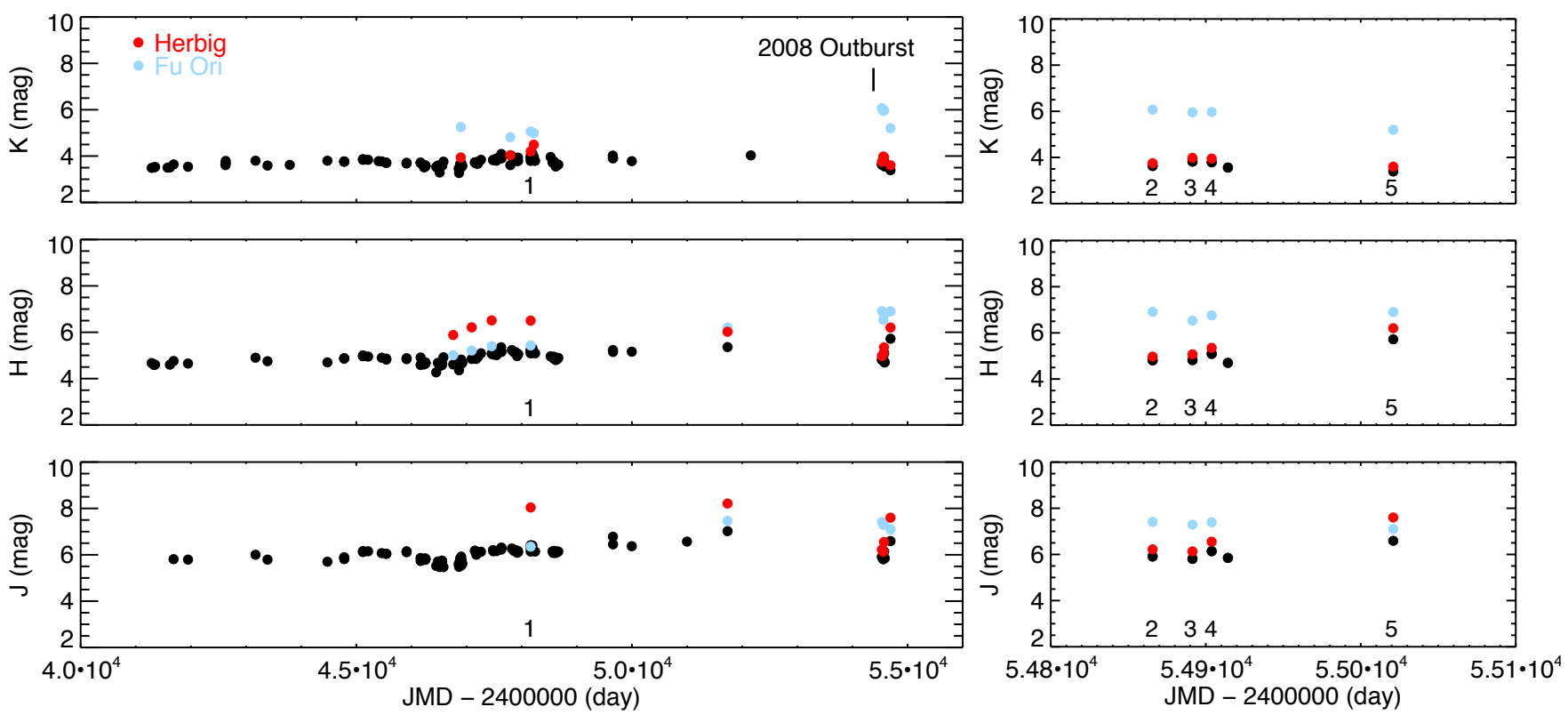

Fig. 4. Resolved Naco and Keck $J H K$ photometry of FUOR component relative to the HBe compared to previous measurements (unresolved components) from the literature (VDA4, Koresko et al. 1991; Haas et al. 1993, and references therein). The epochs with simultaneous $J H K$-band photometry are: (1) October 1990 (quiescence); (2-3-4), January 31, February 26, and March 11, 2009 respectively (outburst); (5) December 2009 (returning to quiescence).

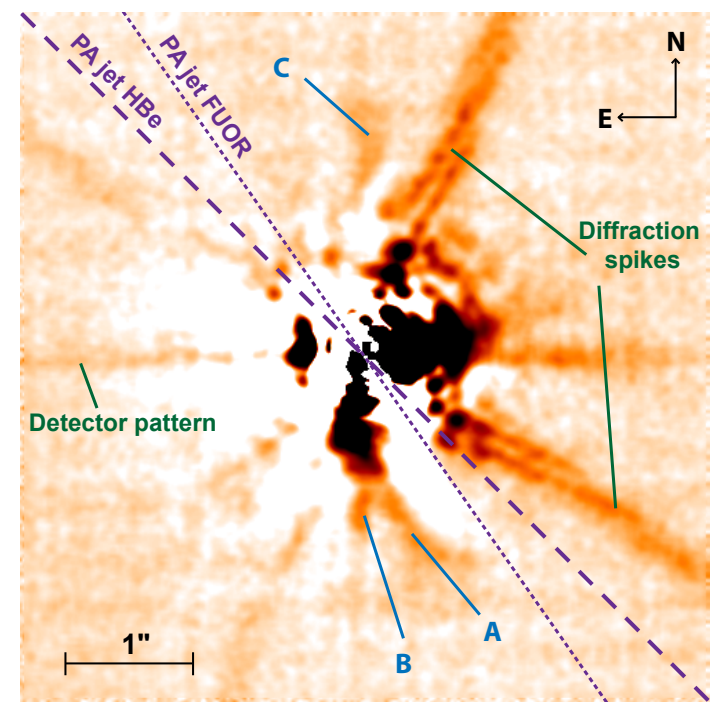

Fig. 5. VLT/NACO PSF-subtracted $L^{\prime}$-band image of Z CMa obtained on January 31, 2009. See Sect. 4.2 for details.

\subsection{Astrometry and orbital motion}

The astrometry of the FUOR relative to the $\mathrm{HBe}$ was measured at each epoch for our $\mathrm{NaCo}$ and Keck data. The results are reported in Table 2 and shown in Fig. 6. They complement older epochs obtained using AO and speckle (Millan-Gabet et al. 2001; Koresko et al. 1991; Haas et al. 1993; Barth et al. 1994; Thiebaut et al. 1995) and more recent measurements obtained with the polarimetric imaging mode of $\mathrm{NaCo}$ and SPHERE (Canovas et al. 2015; Antoniucci et al. 2016). While the separation does not seem to vary significantly, our astrometric measurements confirm a significant variation of position angle since 1986. This might be consistent with a coplanar configuration between the binary and the HBe and FUOR disks (Malbet et al. 1993; Benisty et al. 2010) as often expected in binary systems,

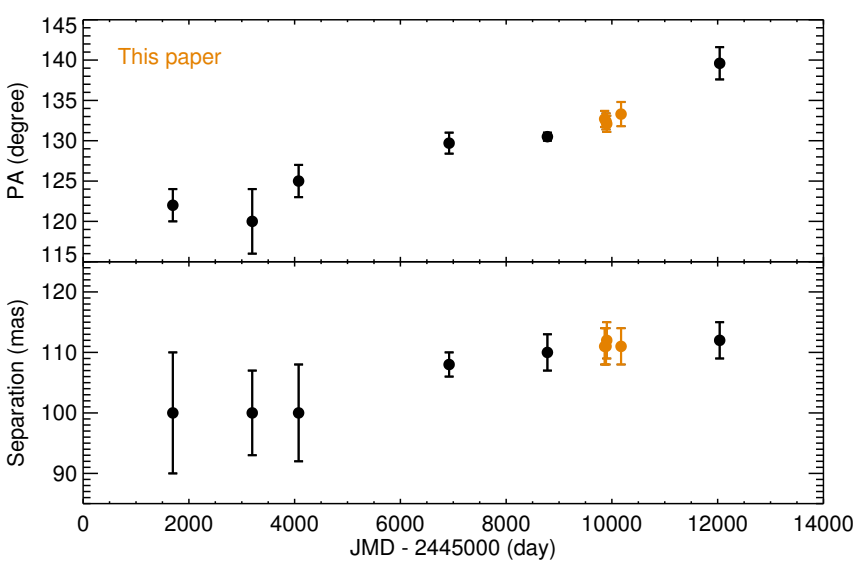

Fig. 6. Position in separation and position angle of the FUOR component relative to the $\mathrm{HBe}$, based on the literature and our $\mathrm{NaCo}$ and Keck observations.

although the jet orientations differ by at least 10 degrees between the two components (Whelan et al. 2010).

\section{Spectro-imagery of the components of Z CMa}

The flux-calibrated SINFONI spectra are shown in the $1.1-2.4 \mu \mathrm{m}$ range in Fig. 7. The OSIRIS and SINFONI spectra of the FUOR and of the HBe are compared in each individual band in Figs. 8-10.

\subsection{Near-infrared spectrum of the HBe component}

The 1.1-2.5 $\mu \mathrm{m}$ spectrum of the HBe component is dominated by a complex set of emission lines overlaid on a continuum with a red slope. These lines are decreased or absent in the OSIRIS spectrum obtained during the quiescent phase. The 
M. Bonnefoy et al.: Multi-epoch images and spectra of the components of Z CMa in the near-infrared during the 2008 outburst

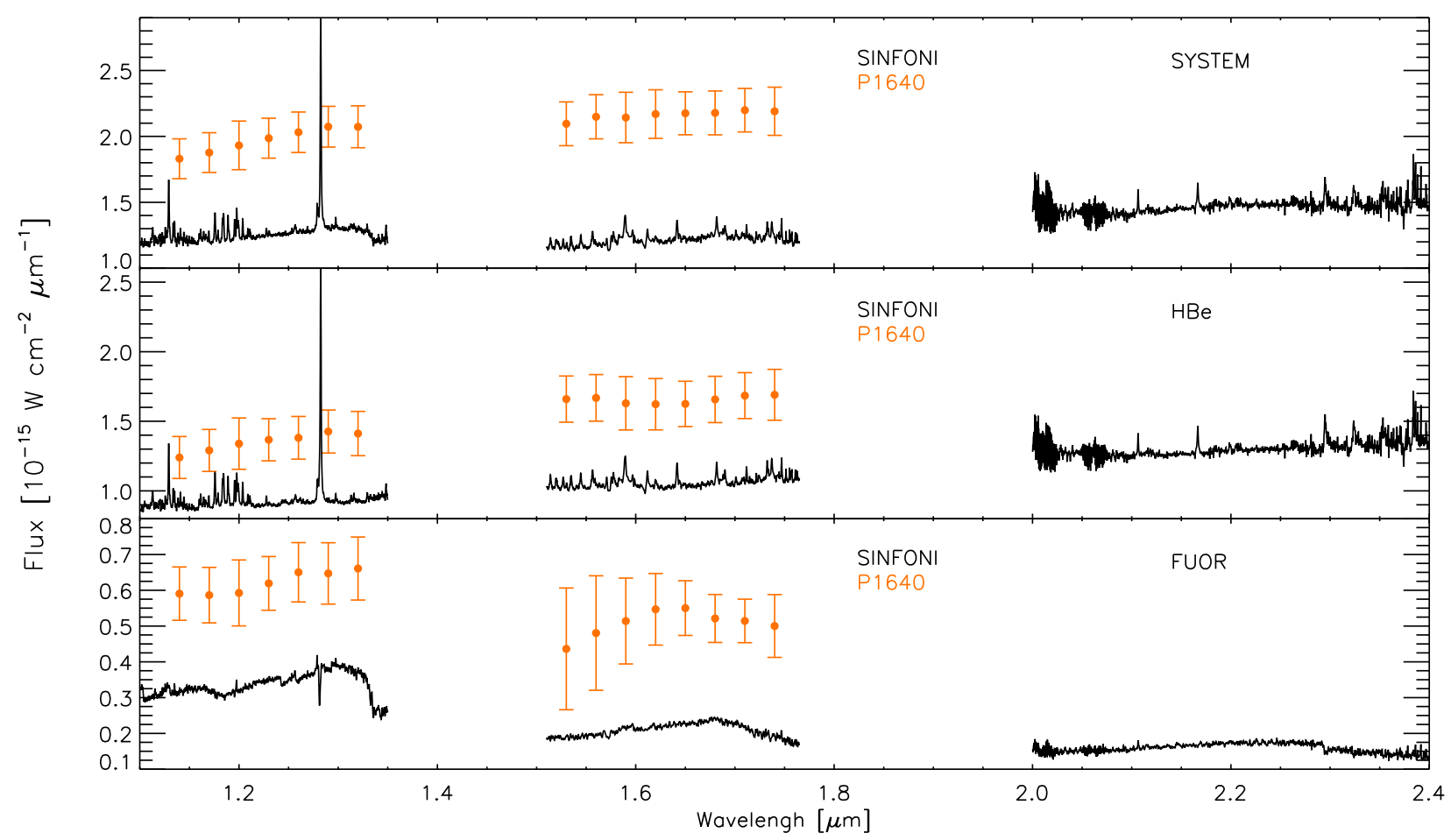

Fig. 7. Flux-calibrated SINFONI spectra (from February 6, 2009) of the system (top), the HBe component (middle), and of the FUOR (bottom) in the 1.1-2.4 $\mu \mathrm{m}$ range while the binary was in outburst. The lower resolution $(R \sim 45)$ P1640 spectrum of the system components also acquired during the outburst phase (March 17, 2009, Hinkley et al. 2013) is shown in orange.

NIR color-variation taken close in time with the OSIRIS spectra indicates that the continuum became redder when the system returned to quiescence. This is consistent with the slope variation of the continuum in the $J$-, $H$-, and $K$-band spectra, although there are some uncertainties on the calibration of this slope in the OSIRIS data (see Sect. 5.2). The position of the emission lines emerging at more than $5 \%$ of the continuum flux are reported in Table C.1. They were retrieved using an interactive least-squares multiple Gaussian fitting tool from the FUSE IDL library ${ }^{2}$ with an accuracy of $\sim 1 \AA\left(12-28 \mathrm{~km} \mathrm{~s}^{-1}\right)$. The velocity of each identified line, their corresponding transition, the associated nondereddened fluxes, full-width at half maximum (FWHM), and equivalent widths (EW) for unblended lines complete Table C.1. The velocities were corrected for the radial velocity of the system $\left(+30 \mathrm{~km} \mathrm{~s}^{-1}\right.$, Hartmann et al. 1989) and for the barycentricto-helocientric velocity shift at the time of the observations $\left(-11.2 \mathrm{~km} \mathrm{~s}^{-1}\right)$. The fluxes were estimated following the estimation and the removal of the continuum beneath the line using a low-order Legendre polynomial. The associated error bar was estimated from the rms of the noise inside the continuum estimation zones surrounding the lines. FWHM are taken from the Gaussian fit. Finally, equivalent widths (EW) and their associated error bars are computed following the Sembach \& Savage (1992) method.

\subsubsection{Line identification}

We propose an identification of most of the lines, for which we rely on analogies between our spectrum and those of several

\footnotetext{
2 The tool can be downloaded at http//fuse.pha.jhu.edu/
} analysis/fuse_idl_tools.html
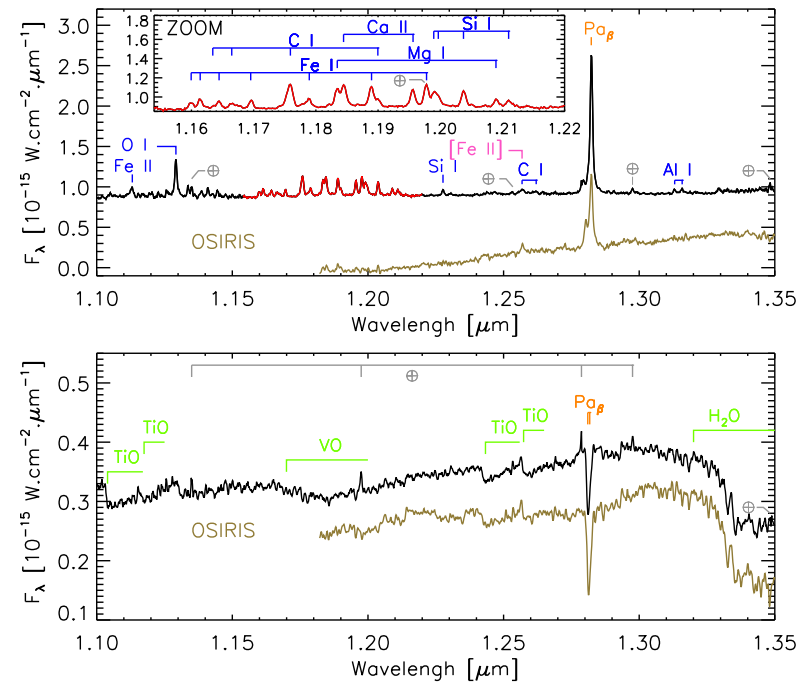

Fig. 8. $J$-band spectra of the Z CMa HBe (top) and FUOR (bottom) components during the outburst phase (black and red line for the zoom; SINFONI spectrum from February 6, 2009) and in the quiescent stage (golden line; OSIRIS spectrum from December 22, 2009). The fluxes of the OSIRIS spectra are normalized to the median flux of the SINFONI spectra between 1.206 and $1.246 \mu \mathrm{m}$ and shifted to lower values for clarity.

young embedded targets found in the literature (Kelly et al. 1994; Greene \& Lada 1996; Nisini et al. 2005; Gibb et al. 2006; Antoniucci et al. 2008; Connelley \& Greene 2010; Covey et al. 2011; Kóspál et al. 2011b; Caratti o Garatti et al. 2013; Cooper et al. 2013). We completed and double-checked the identification using three databases for atomic and ionic 

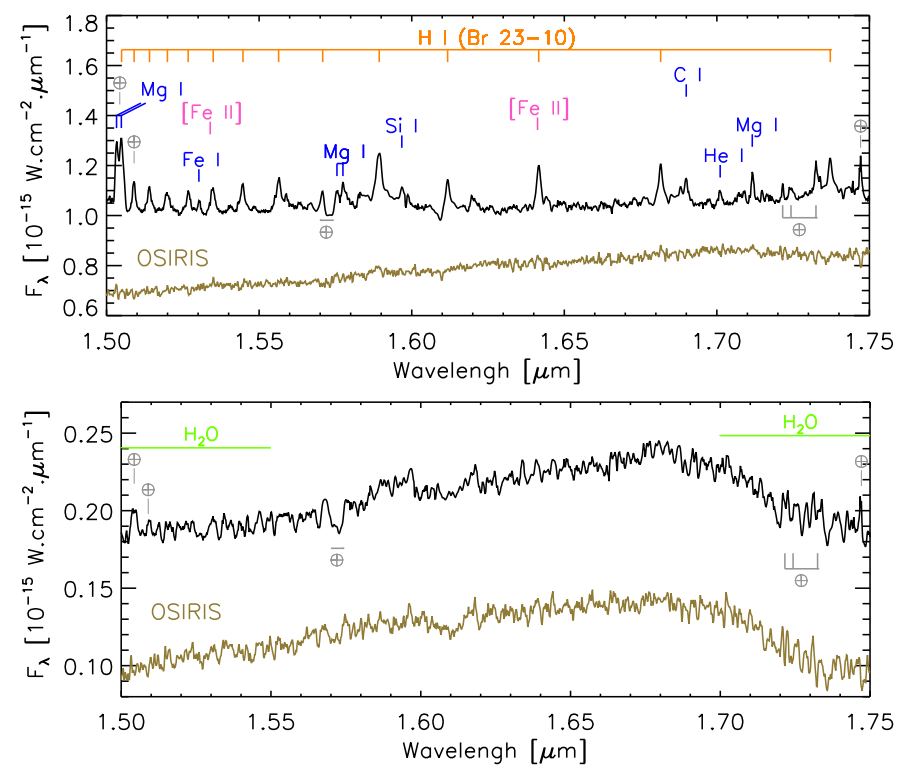

Fig. 9. $H$-band spectra of the Z CMa HBe (top) and FUOR (bottom) components during the outburst phase (black line; SINFONI spectrum from February 6, 2009) and in the quiescent stage (golden line; OSIRIS spectrum from December 22, 2009). The fluxes of the OSIRIS spectra are normalized to the median flux of the SINFONI spectra between 1.66 and $1.70 \mu \mathrm{m}$ and shifted to lower values for clarity.
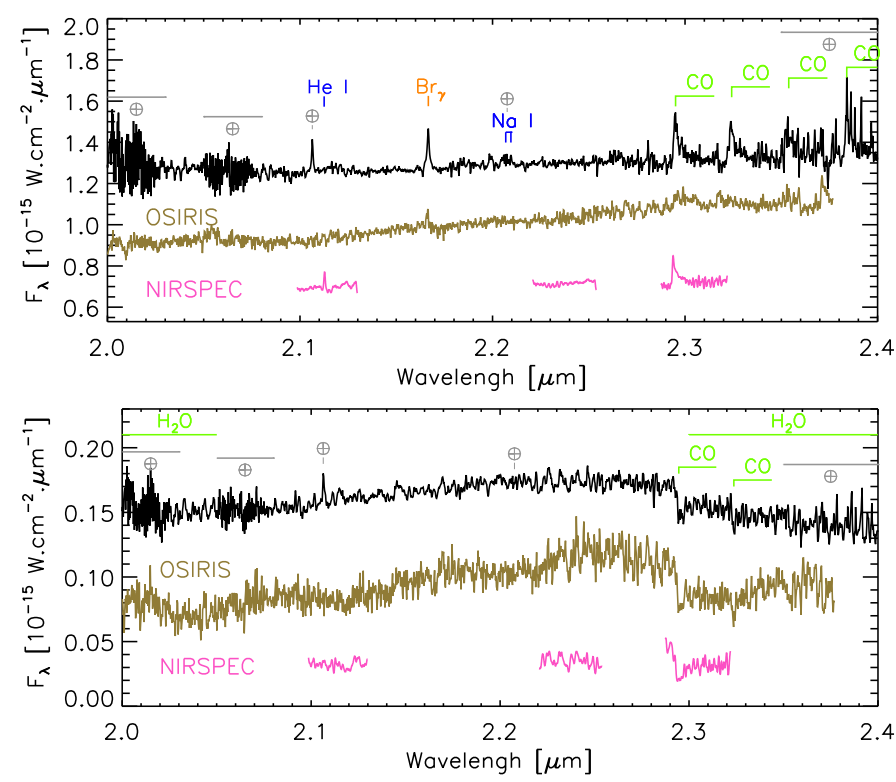

Fig. 10. Same as Fig. 9 but for the $K$ band. The normalized NIRSPEC spectra (with respect to the continuum of the SINFONI spectra) of the system components obtained while the binary was in a quiescent state (December 17, 2006) are overlaid in pink. The fluxes of the OSIRIS spectra are normalized to the median flux of the SINFONI spectra between 2.18 and $2.22 \mu \mathrm{m}$ and shifted to lower values for clarity.

transitions $s^{3,4,5}$. We also carefully flagged spurious lines that were introduced while dividing the spectrum by the telluric standards.

\footnotetext{
3 http://physics.nist.gov/PhysRefData/ASD/lines_form. html

4 http://www.pa.uky.edu/\$ \$sim\$peter/atomic/

5 http://www.cfa.harvard.edu/amp/ampdata/kurucz23/ sekur.html
}
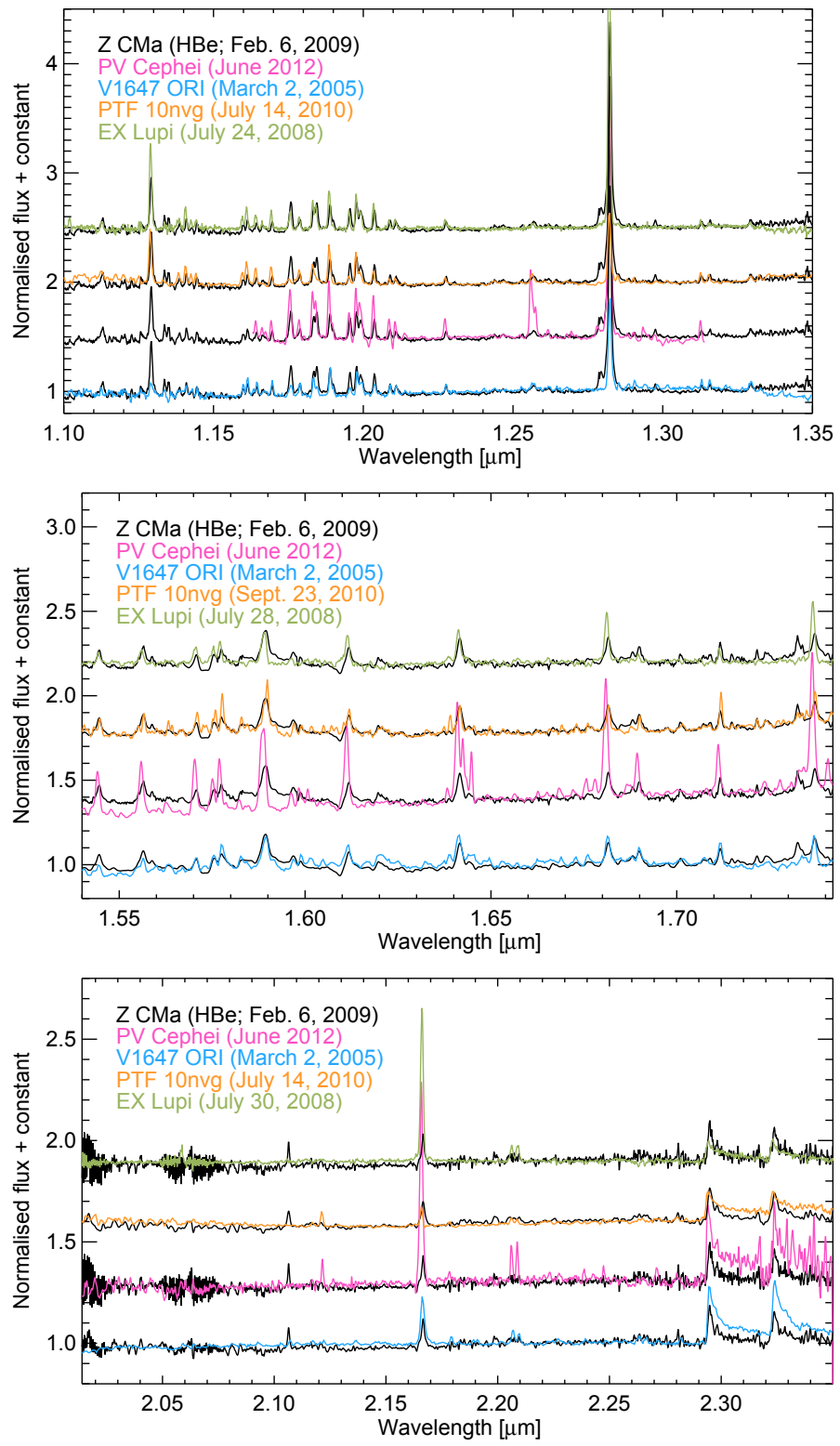

Fig. 11. Comparison of the NIR spectrum of the HBe component of Z CMa to spectra of variable young stellar objects PV Cephei (Caratti o Garatti et al. 2013), V1647 ORI (Gibb et al. 2006), PTF 10nvg (Covey et al. 2011; Hillenbrand et al. 2013), and EX Lupi (Kóspál et al. 2011b). All spectra were smoothed to the lowest common resolution. Spectra of PV Cephei, V1647 ORI, and PTF 10nvg were dereddened by $A_{V}=8,9$, and 6 mag, respectively to reproduce the Z CMa's HBe pseudo-continuum slope. The $H$-band spectrum of EX Lupi is dereddened by $A_{V}=1$, while the $K$-band spectrum is redenned by $A_{V}=1$.

We retrieved the same emission lines in the EX Or prototype EX Lup captured during an outburst (Kóspál et al. 2011b). The spectrum also resembles those of the outbursting protostars V1647 Ori, PTF 10nvg, and PV Cephei (Gibb et al. 2006; Covey et al. 2011; Hillenbrand et al. 2013; Caratti o Garatti et al. 2013, see Fig. 11). We also find a good match with those of class I stars IRAS $03220+3035$ N, IRAS $16289-4449$, and IRAS $20453+6746$ (Connelley \& Greene 2010), and of the embedded R CrA objects IRS2 and HH100 IR (see Figs. 2-4 of Nisini et al. 2005). 
The spectrum is dominated by hydrogen emission lines. The Paschen $\beta$ represents the strongest line of the spectrum. In the $H$ band, the more remarkable emission features are the series of Brackett lines (order 10 to 22). The Brackett 23 line is expected to be blended with a Mg I emission feature at $1.504 \mu \mathrm{m}$. The $K$ band also has a moderate $\mathrm{Br} \gamma$ emission line (also present in the AMBER spectra of the HBe, Benisty et al. 2010).

The $J$-band spectrum is also dominated by an $\mathrm{O}$ I line at $1.1292 \AA$. The O I line is commonly attributed to fluorescence excitation by a UV continuum and/or to resonant absorption of Ly $\beta$ photons (Kelly et al. 1994; Nisini et al. 2005). The nondetection of the $\mathrm{O}$ I line at $1.316 \mu \mathrm{m}$ favors the second hypothesis (Kóspál et al. 2011b).

A forest of lines is also present from 1.15 to $1.22 \mu \mathrm{m}$. This forest most likely corresponds to the broad feature seen in the spectra of HH100 IR and R Cra IRS2. It is composed of permitted lines of the neutral species of $\mathrm{Fe}, \mathrm{C}, \mathrm{Si}, \mathrm{Mg}$, and of inonized Ca. We also identify other lines of Fe I, Si I , C I, Al I, Na I, and $\mathrm{Mg}$ I at longer wavelengths in the $J, H$, and $K$ bands.

Only one ionized species can be firmly identified in the spectrum (Ca II). The first ionization potential of $\mathrm{Ca}(6.11 \mathrm{eV})$ is lower than those of $\mathrm{Si}(8.15 \mathrm{eV})$ but remains comparable or higher than those of $\mathrm{Mg}(7.64 \mathrm{eV}), \mathrm{Al}(5.98 \mathrm{eV})$ and $\mathrm{Na} \mathrm{I}$ $(5.14 \mathrm{eV})$. The absence of $\mathrm{Al}$ II, Na II, and $\mathrm{Mg}$ II emission can be explained by the energies of the upper transition levels $(12.1-37.2 \mathrm{eV})$ in the $1.1-2.5 \mu \mathrm{m}$ range. These energies are significantly higher than those involved for the Ca II $(7.5 \mathrm{eV})$. As noted by Kóspál et al. (2011b), the ionization potentials of the metallic species are lower $(5.14-8.15 \mathrm{eV})$ than the one of carbon $(11.26 \mathrm{eV})$, oxygen $(13.62 \mathrm{eV})$, and hydrogen $(13.6 \mathrm{eV})$. This suggests that metals lies in a medium where hydrogen is mostly neutral.

We report a weak [Fe II] emissions around $1.257 \mu \mathrm{m}, 1.534$, and $1.644 \mu \mathrm{m}$ with several velocity components, associated with a micro jet studied in Whelan et al. (2010). The $1.644 \mu \mathrm{m}$ line is partially blended with the $\mathrm{Br}_{12}$ line. We analyze structures associated with the $1.534 \mu \mathrm{m}$ line in Sect. 5.3.

The spectrum has strong ${ }^{12} \mathrm{CO}$ overtones of the $X^{1} \Sigma^{+}-$ $X^{1} \Sigma^{+}$system seen in emission in the $K$ band. These overtones have previously been reported by Hinkley et al. (2013), while the system was in the quiescence phase before the 2008 outburst. This is retrieved in many young stellar objects (e.g., Connelley \& Greene 2010; Cooper et al. 2013). Connelley \& Greene (2010) showed that Bry coincides with the emergence of the $\mathrm{CO}$ band-head seen in emission. Since $\mathrm{Br} \gamma$ is a well-known accretion tracer (Muzerolle et al. 1998), the authors proposed that the $\mathrm{CO}$ emission might arise from disk surfaces when the systems are quite veiled and accretion rates are high. We follow this hypothesis and model them in Sect. 5.1.4. Alternatively, $\mathrm{CO}$ overtones might be produced inside magnetospheric accretion funnels (Martin 1997). This line is not detected in the spectrum that Liljeström \& Olofsson (1997) obtained while the system was in quiescence.

To conclude, the comparison of the HBe spectrum to the one of PTF 10nvg during its brighter stages suggests that we may also be seeing water-band emission in the spectrum of the $\mathrm{HBe}$ at $1.33-1.35 \mu \mathrm{m}, 1.49-1.55 \mu \mathrm{m}$, and $1.70-1.75 \mu \mathrm{m}$.

\subsubsection{Line profiles}

Most of the lines have a FWHM greater than the instrumental line-width (5.11 $\AA$ in the $J$ band, $5.00 \AA$ in the $H$ band, $3.68 \AA$ in the $K$ band; measured on thorium-argon calibration lines). The
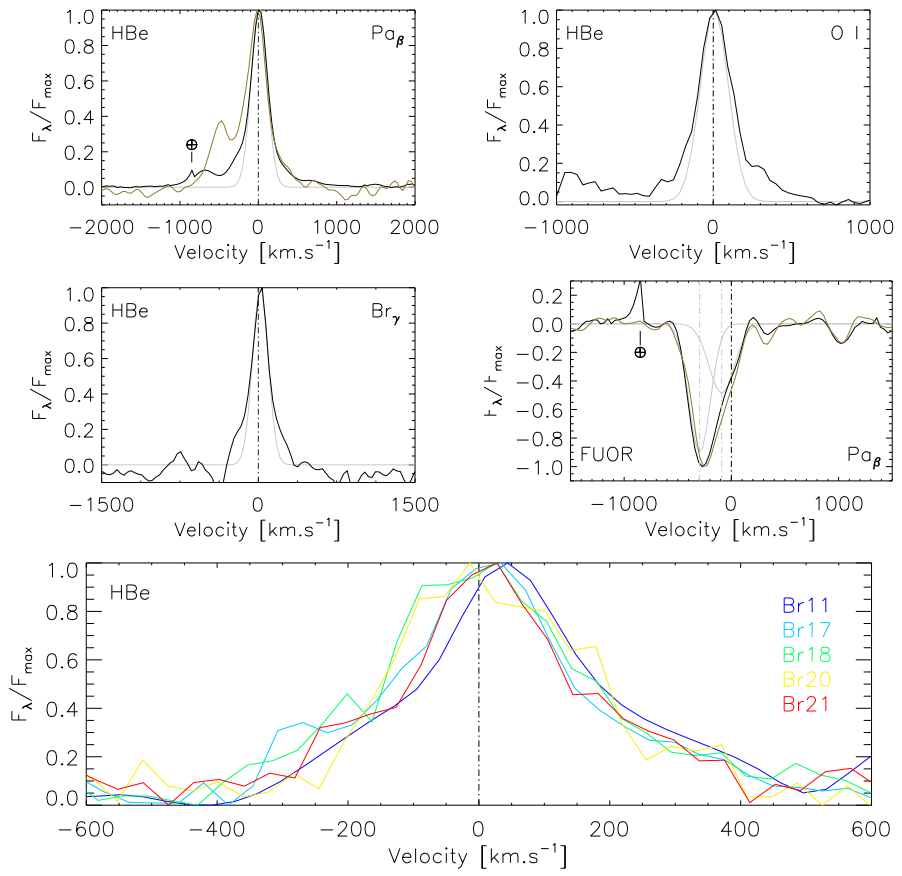

Fig. 12. Profile of strong and weakly blended lines for the $\mathrm{HBe}$ and FUOR components. The black solid lines correspond to the SINFONI (outburst) data, while the golden lines correspond to the OSIRIS spectra (post-outburst).

profiles of the $\lambda 1.1292 \mathrm{O} \mathrm{I}, \mathrm{Pa} \beta$, and of several other Brackett lines that are unaffected by a strong blend $(\mathrm{Br} \gamma, \mathrm{Br} 11,17,18$, 20, and 21) are reported in Fig. 12. They were subtracted from their continuum and normalized to their highest value. The profiles of the $\mathrm{Pa} \beta$ lines extracted from the SINFONI (black line) and OSIRIS (golden line) spectra are compared in the upper left panel. A Gaussian is fitted on the line core and is overlaid (gray line).

The oxygen line core has a non-significant velocity. The line has two broad asymmetrical wings that extend up to -400 and $+600 \mathrm{~km} \mathrm{~s}^{-1}$. An additional unrelated feature (possibly an Al I line) is present at $-900 \mathrm{~km} \mathrm{~s}^{-1}$.

The resolved profiles of isolated $\mathrm{H}$ I emission lines $(\mathrm{Pa} \beta$, $\mathrm{Br} 11, \mathrm{Br} 17, \mathrm{Br} 18, \mathrm{Br} 20$, and $\mathrm{Br} 21)$ are shown in Fig. 12. The $\mathrm{Pa} \beta$ profile is divided into a main component characterized by asymmetrical wings and into a blueshifted lobe at $-650 \mathrm{~km} \mathrm{~s}^{-1}$. The lobe appears at lower velocities $\left(-480 \mathrm{~km} \mathrm{~s}^{-1}\right)$ in the OSIRIS spectrum and contributes more to the total line flux. We retrieved these two components in the spectrum of EX Lup (Kóspál et al. 2011b), PV Cephei (Caratti o Garatti et al. 2013), and PTF 10nvg (Covey et al. 2011; Hillenbrand et al. 2013). The velocity is consistent with an emission coming from the basis of the HBe jet (Whelan et al. 2010). The line peak is slightly redshifted $\left(42 \mathrm{~km} \mathrm{~s}^{-1}\right)$, as is the one of PV Cephei (Caratti o Garatti et al. 2013). The profile also has a red tail that extends up to $\sim 1000 \mathrm{~km} \mathrm{~s}^{-1}$ with a bump at $+700 \mathrm{~km} \mathrm{~s}^{-1}$. We retrieved this long tail in the $\mathrm{H} \alpha$ and $\mathrm{H} \beta$ lines seen in the 1996 and 2000 spectra of Z CMa (van den Ancker et al. 2004). VDA04 proposed that the tail originates from the extended atmosphere of the HBe. The bump is very consistent with the one seen in the spectrum of EX Lup during outburst (Kóspál et al. 2011b). In summary, the complex profile of Paschen $\beta$ (and related time variations) indicates that the line forms in (at least) two distinct regions: one at the basis of the ouflow, and another closer to the star. 


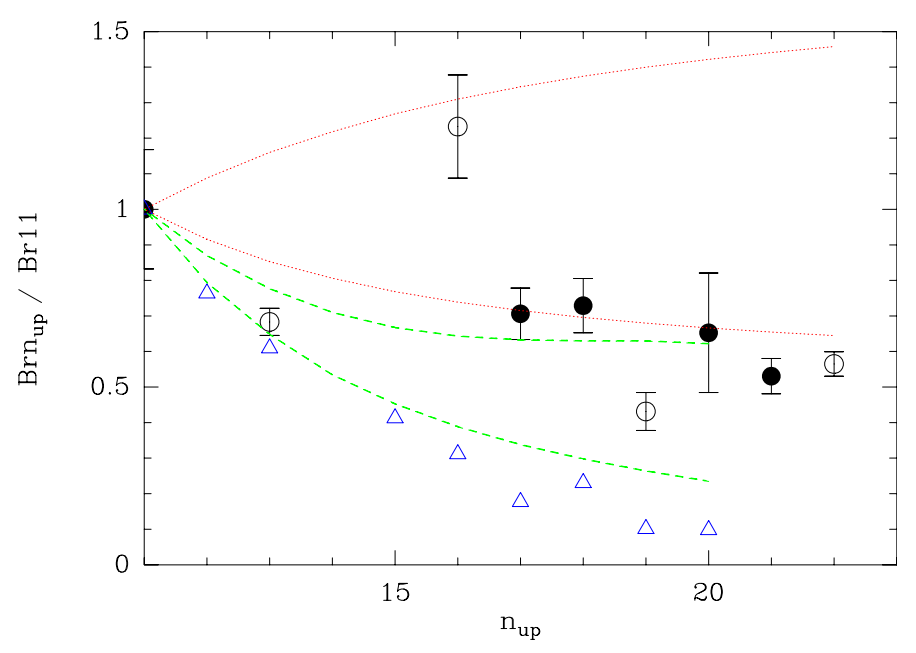

Fig. 13. $H$ band $H I$ Brackett excitation diagram for the HBe of ZCMa (circles). All line fluxes are normalized to the HI Brackett 11 line. Open circles show the lines that are probably contaminated. Blue triangles show the line ratios detected in EX Lupi (Kóspál et al. 2011b). The green curves are predictions from case B recombination from Hummer \& Storey (1987) for $T=10^{4} \mathrm{~K}$ and $n=10^{8} \mathrm{~cm}^{-3}$ (lower curve) and for $T=500 \mathrm{~K}$ and $n=10^{7} \mathrm{~cm}^{-3}$ (upper curve). The red dotted lines show expected line ratios from blackbody emission at $T=1000 \mathrm{~K}$ (lower curve) and $T=10^{4} \mathrm{~K}$ (upper curve).

Brackett lines in the $H$ band are slightly redshifted at the peak $\left(+25 \mathrm{~km} \mathrm{~s}^{-1}\right)$ and have a symmetrical profile extending to $\pm 400 \mathrm{~km} \mathrm{~s}^{-1}$. The Br $\gamma$ profile is slightly asymmetric and redshifted. This profile is coherent with the double-peaked asymmetric profile (peaks at $\sim-40$ and $120 \mathrm{~km} \mathrm{~s}^{-1}$ ) studied at $R \sim$ 12000 while the system was in outburst by Benisty et al. (2010). We showed that the $\mathrm{Br} \gamma$ line originates from a bipolar wind at the au scale. The same is expected for the other lines of the series.

\subsubsection{H I line ratio}

We show in Fig. 13 the HI line ratios observed during the photometric outburst phase for the HBe component. We only include the HI Brackett lines detected in the $H$ band to overcome the effect of poorly constrained reddening correction. The HI excitation diagram is significantly flatter than observed in EX Lupi (Kóspál et al. 2011b) and in other UX Ori outbursting stars (Lorenzetti et al. 2009). Both optically thin case-B recombination and optically thick blackbody emission can reproduce the observed line ratios but would require very low gas temperatures $(500-1000 \mathrm{~K})$ and moderate densities of $10^{7}-10^{8} \mathrm{~cm}^{-3}$, suggesting that the lines do not originate in the immediate vicinity of the star. Such temperatures and densities could be found in the surface layers of the accretion disk, which are strongly irradiated by the central star and accretion luminosity, or at the base of the outflow. We derive a lower limit to the $\mathrm{Pa} \beta / \mathrm{Br} \gamma$ ratio of 7.7 without reddening correction. For the $\mathrm{Pa} \beta$ line, we integrated the emission in the same velocity range as observed in $\mathrm{Br} \gamma$, that is between -300 and $+300 \mathrm{~km} \mathrm{~s}^{-1}$. This very high ratio is marginally compatible with case-B recombination and would exclude optically thick blackbody emission. The limit for optically thick blackbody emission is $\mathrm{Pa} \beta / \mathrm{Br} \gamma \leq 4$ (Antoniucci et al. 2011). However, it is not clear that the $\mathrm{Pa} \beta$ and $\mathrm{Br} \gamma$ lines originate from the same regions as they show both distinct emission profiles (Sect. 5.1.2), and different behaviors with time (Sect. 5.1.5). It is therefore likely that simple models assuming constant density and temperature are not an

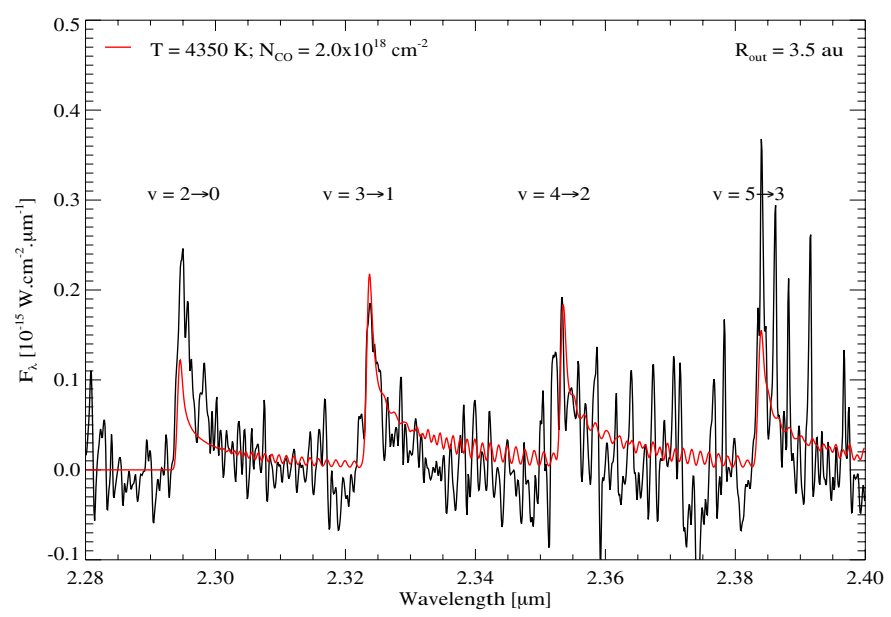

Fig. 14. $\mathrm{CO}$ bandhead seen in the SINFONI spectrum of the $\mathrm{HBe}$ (black) compared to our best-fit model for an outer radius of the lineemitting region of $3.5 \mathrm{au}$ (red).

adequate representation of the HI-emitting medium. Models of spherically expanding wind in LTE predict both high $\mathrm{Pa} \beta / \mathrm{Br} \gamma$ ratios and flat $\mathrm{HI}$ excitation diagrams (Lorenzetti et al. 2009; Antoniucci et al. 2011). The observed $\mathrm{Pa} \beta$ flux and $\mathrm{Pa} \beta / \mathrm{Br} \gamma$ ratio would require mass-loss rates $\geq 10^{-6} M_{\odot} \mathrm{yr}^{-1}$ and an envelope thickness $>100 R_{\odot}$. Full radiative transfer modeling in either a disk or outflow model would be required, but this is beyond the scope of this paper.

\subsubsection{Modeling of the $\mathrm{CO}$ emission lines}

The $\mathrm{CO}$ overtones seen in emission in the $\mathrm{HBe}$ component are also a characteristic feature of other EX Or (see Fig. 11). Eisner et al. (2014) have used interferometry to resolve the CO line emitting areas around Herbig stars. They found that the regions of the disks emitting in the $\mathrm{CO}$ bandhead lines are located between 0.05 and 2 au from the central star. We therefore modeled the $\mathrm{CO}$ lines of the $\mathrm{HBe}$ spectrum acquired in outburst following a similar approach as Kóspál et al. (2011b) for EX Lupi. The $\mathrm{CO}$ disk emission model and related fitting procedure are described in Appendix A. We conclude that the $\mathrm{CO}$ gas in $\mathrm{Z} \mathrm{CMa}$ has an excitation temperature of about $4300 \pm 100 \mathrm{~K}$, and the $\mathrm{CO}$ column density is between $2 \times 10^{18} \mathrm{~cm}^{-2}$ and $2 \times 10^{19} \mathrm{~cm}^{-2}$, depending on the disk size. Combinations of the outer radius of the emitting disk region $R_{\text {out }}$ and of the CO column density $N_{\mathrm{CO}}$ can give similar fit. We show in Fig. 14 the best-fitting model for $R_{\text {out }}=3.5$ au.

We therefore conclude that the $\mathrm{CO}$ emission can be reproduced to first order by the emission from a disk model. The excitation temperature is almost $2000 \mathrm{~K}$ hotter than the one derived for EX Lup by Kóspál et al. (2011b) with the same disk modeling procedure.

\subsubsection{Spectro-photometric variability}

We report in Fig. 1 the dates of the spectroscopic observations of the HBe on the light curve of the system.

The low-resolution spectrum $(R \sim 250)$ of the system gathered during ourburst by Antoniucci et al. (2009) also displays the $\mathrm{O} \mathrm{I}$, the Paschen $\beta$ emission line, and the Brackett lines seen in the SINFONI $H$-band spectrum of the HBe (Anto+09 in Fig. 1). The comparison of the SINFONI (this work) and 

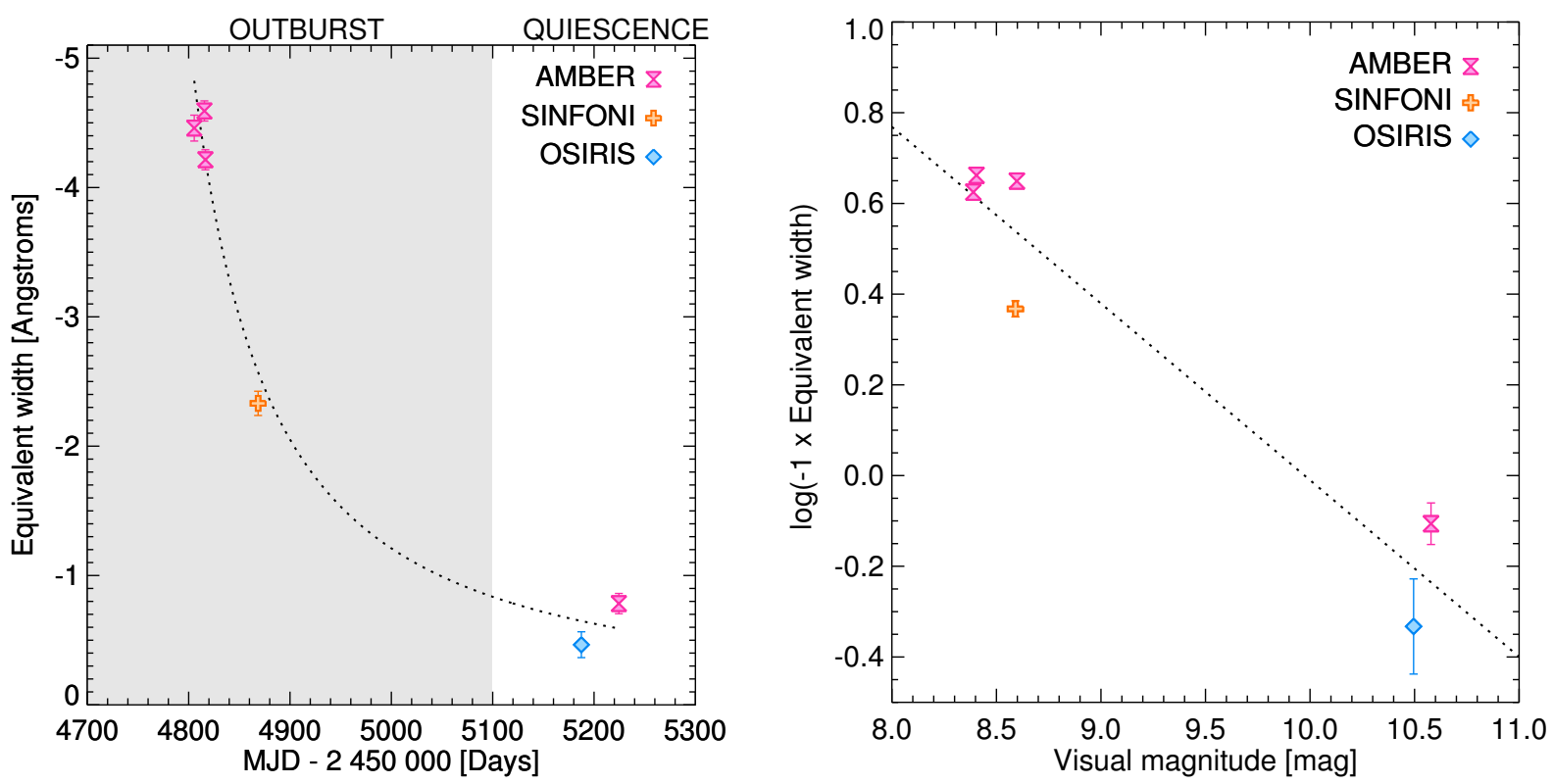

Fig. 15. Decline of the $\operatorname{Br} \gamma$ equivalent width with time (left) and with the system $V$-band magnitude (right).

P1640 (Hinkley et al. 2013) spectra obtained during outburst, the OSIRIS spectrum (while the system was returning to quiescence; this work), and the pre-outburst (quiescent state) NIRSPEC spectrum from 2006 (Hinkley et al. 2013) enable us to pinpoint the evolution of the features related to the HBe during outburst. This collection of data is complemented by a low-resolution $(R \sim 690) K$-band spectrum of each component from November 1992 (Liljeström \& Olofsson 1997) while the system was in quiescence, but still at a magnitude lower than the one of 2006 and 2009. The evolutions of the equivalent widths of the $\mathrm{Pa} \beta$, $\mathrm{Br} \gamma$, and $v=2 \longrightarrow 0{ }^{12} \mathrm{CO}$ are reported in Table C.2.

The evolution of the $\mathrm{Pa} \beta$ line (Fig. 12) of the HBe shares some behavior with the one of PV Cephei. The blueshifted lobe that is seen in absorption in the spectrum of PV Cephei (P Cygni profile) only appears in the ourburst phase. The equivalent widths of the lines for the HBe and this object decreased by $\sim 50 \%$ in 10 and 9 months, respectively, after the outburst. The decline of the equivalent width is consistent with the one of the $v=2 \longrightarrow 0{ }^{12} \mathrm{CO}$ overtone.

In Fig. 15, we show that the equivalent width of the $\operatorname{Br} \gamma$ line decreases after the photometric outburst. This decline can be reproduced by the function $E W(t)=A /(t-B)^{\beta}$, where $A$ and $B$ are constant values modulating the amplitude and epoch of maximum outburst, and $\beta$ the decline factor. We used a LevenbergMarquardt fitting tool to estimate $A=-1516 \pm 1828, B=$ $4708 \pm 25$, and $\beta=1.25 \pm 0.20$. The $B$ parameter corresponds to an epoch of maximum outburst on August 30, 2008. Unfortunately, this falls at a time when the $V$ band was not monitored, but the system was considered to be in outburst at that time. We caution of course that the analysis is limited by the small number of epochs. The equivalent widths of the line decline with that of the $V$-band magnitude of the system (correlation factor $=0.95$ ) taken from the AAVSO International database ${ }^{6}$ (Kafka 2016). This line is not detected in the November 1992 low-resolution spectrum of the $\mathrm{HBe}$, which confirms that it is related to the system activity.

\footnotetext{
6 http://www . aavso.org
}

The NIRSPEC spectrum obtained while the system was in a quiescent state shows a He I line $\left(2.1125 \mu \mathrm{m}, 3 \mathrm{p}^{3} \mathrm{P}^{\circ}-4 \mathrm{~s}^{3} \mathrm{~S}\right)$. This line is not retrieved in the SINFONI and OSIRIS spectra. It is seen in some YSO spectra and is commonly associated with the stronger $2.0508 \mu \mathrm{m}$ He I line $\left(2 \mathrm{~s}^{1} \mathrm{~S}-2 \mathrm{p}^{1} \mathrm{P}^{\circ}\right)$ (e.g., Cooper et al. 2013; Murakawa et al. 2013) which has been detected by van den Ancker et al. (2004) in the IRTF spectrum of the system taken when it was returning to quiescence (post2000 outburst). This second line is located outside of the NIRSPEC coverage, but it is still missing from our SINFONI and OSIRIS spectra of the HBe. Cooper et al. (2013) proposed that these lines form by collision-induced excitation in a wind. The disppearance of this line system in the outburst (SINFONI) and post-outburst (OSIRIS) state might be due to the evolving contrast with the continuum emission, or to the variable extinction in the line of sight (see below).

The NIR broadband photometry is mostly sensitive to the continuum emission. Therefore, the decrease of the lineequivalent width coupled to the stagnation ( $K$ band) or decrease ( $V$ band, $J$ and $H$ bands) of the continuum flux indicates that the flux produced by the excited regions does decline as well or that the absorption increases. The bluing of the slope of the HBe in the outburst phase supports the hypothesis of a decrease in extinction as well as an increase of the internal heating of the inner portions of the disk due to an increase in accretion rate. We note that the continuum slope of the SINFONI spectrum is consistent with the one obtained with P1640. Both the SINFONI and OSIRIS $K$-band spectral continua look redder than the one obtained in November 1992 (Fig. 1 of Liljeström \& Olofsson 1997) while the system was in quiescence.

The evolution of the HBe in color-color and color-magnitude diagrams (Fig. 16) indicates that the photometry of the star has one component that follows the interstellar reddening vector. This suggests that the outburst is at least partly due to a reduced extinction in the line of sight. van den Ancker et al. (2004) reported a spectral type B0IIIe for the HBe. Assuming that these stars have NIR photometric colors close to 0 , the location of the $\mathrm{HBe}$ in the Fig. 16 indicates that the star was extincted by $A_{v}=12$ mag during the 2008 outburst. This is consistent 

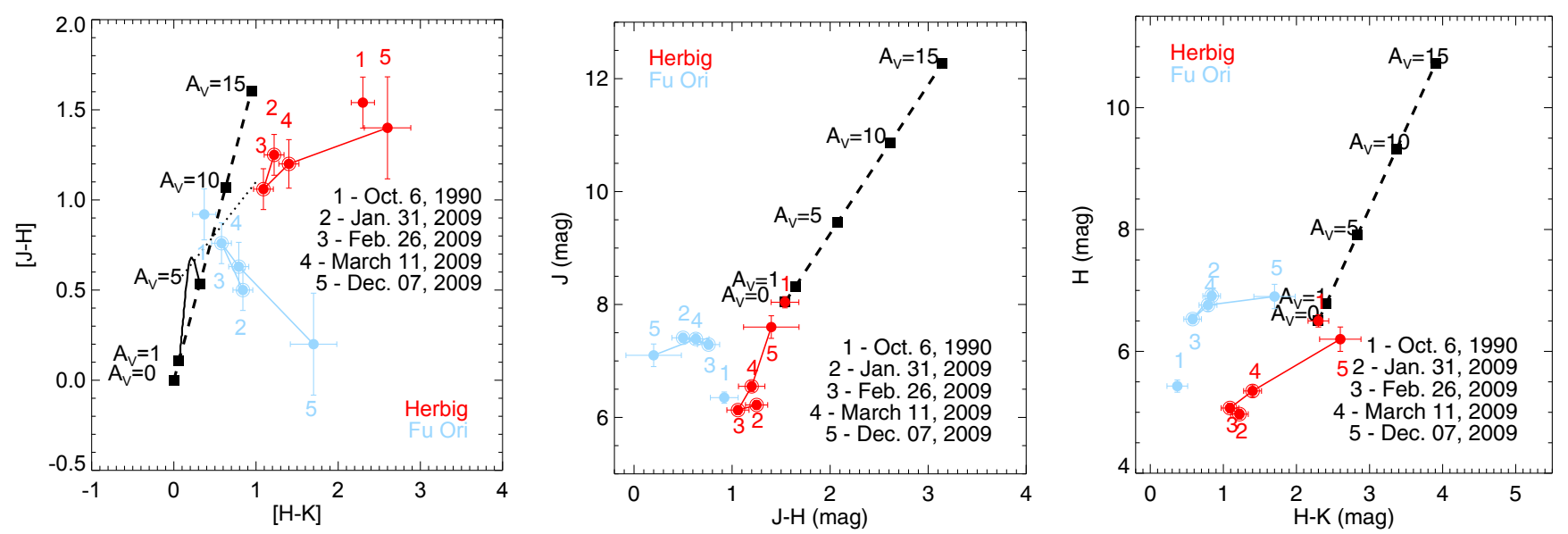

Fig. 16. Evolution of the FUOR and HBe NIR colors and photometry when Z CMa was in a quiescent state (1; October 1990 observations), in outburst (2-3-4; encircled disks; corresponding to NaCo observations on January 31, February 26, and March 11, 2009 respectively) and returning to a quiescent state in December 2009 (5). The reddening vector for visual extinctions of 0, 1, 5, 10, and 15 mag as well as the colors of pre-mainsequence stars (solid line, Pecaut \& Mamajek 2013) and the locus of CTTS (dashed line, Meyer et al. 1997) are overlaid for comparison.

with $A_{v}=10$ mag measured by Hinkley et al. (2013) from the SED analysis of the star obtained during the outburst. This also agrees with the lower limit on $A_{v}$ derived from line ratio in Sect. 5.1.3. The reduced $A_{v}$ found by Hinkley et al. (2013) is also consistent with the higher flux level of their spectra compared to SINFONI's and shown in Fig. 7, although we cannot exclude that the different flux levels may come from differences in the flux-calibration methods used the two sets of spectra.

The variation in $H-K$ color of the HBe component shows an additional contribution perpendicular to the reddening vector. This deviation is reproduced by several EX Ors (Lorenzetti et al. 2006; Kóspál et al. 2011a; Lorenzetti et al. 2012), and in particular EX Lup. EX Lup represents the NIR spectrum of the HBe well (Fig. 11). Lorenzetti et al. (2012) showed that during photometric outbursts of EX Ors, an additional blackbody component appears with temperatures between 1000 and $4500 \mathrm{~K}$ and a flux corresponding to blackbody emission from a uniform disk of radius between 0.01 and 0.1 au. Hinkley et al. (2013) fit the SED of the HBe with two blackbodies at $T_{\text {eff }}=8500$ and $1100 \mathrm{~K}$ behind $A_{V}=10$. But their analysis relies on photometric points obtained while the system was at different stages. As shown in Fig. 3 of Giannini et al. (2016b), an increase in temperature of the blackbody component in the SED of the HBe during the outburst phase might well explain the variation in colors perpendicular to the interstellar reddening vector.

\subsection{FUOR component}

The NIR spectrum of the FUOR component of $\mathrm{Z} \mathrm{CMa} \mathrm{is}$ characteristic of M8-M9 giants of the IRTF spectral library (Rayner et al. 2009), as expected for FU Orionis objects (see Hartmann \& Kenyon 1996) with a luminosity in NIR totally dominated by the accretion disk flux and with the cooler regions of the disk producing deep molecular absorptions. Strong broad absorption bands of $\mathrm{H}_{2} \mathrm{O}$, TiO, $\mathrm{VO}$, and $\mathrm{CO}$ lines are therefore detected. The CO lines in absorption of the FUOR were previously marginally detected in the unresolved spectrum of $\mathrm{Z} \mathrm{CMa}$ by Reipurth \& Aspin (1997), through the strong contribution of the HBe component the $K$-band flux. These absorptions are typical of FU Orionis objects (Mould et al. 1978; Carr et al. 1989). The individual spectrum of the component obtained by Liljeström \& Olofsson (1997) and Hinkley et al. (2013) showed that the $\mathrm{CO}$ in absorption can be associated with this component. The SINFONI (outburst) and OSIRIS (returning to quiescence) spectra shown in Figs. 8-10 unambiguously confirm this result.

The OSIRIS and SINFONI spectra display a strong $\mathrm{Pa} \beta$ absorption. This line is retrieved in the spectra of FU Orionis objects HBC687 (=IRAS 19266+0932) and V1735 Cyg (=IRAS $21454+4718)$ of the Connelley \& Greene (2010) sample. The overall spectrum of HBC687 better reproduces the slope of the FUOR component of Z CMa.

The profile of the Paschen $\beta$ absorption can be fit by two Gaussian functions with $F W H M$ of 8 and $11.5 \AA$ (broadly consistent with the spectral resolution), intensity ratio beneath the pseudo-continuum of 1.5 , and velocities of $-265 \mathrm{~km} \mathrm{~s}^{-1}$ and $-102 \mathrm{~km} \mathrm{~s}^{-1}$, respectively. This double-peaked profile is characteristic of FU Orionis stars in the optical (e.g., Hartmann \& Kenyon 1985). It is reminiscent of the $\mathrm{H}_{\gamma}$ and $\mathrm{H}_{\delta}$ line profiles extracted from the optical spectrum of $\mathrm{Z} \mathrm{CMa}$ acquired in February 1983, while the system was not in outburst (Covino et al. 1984). The spectra of HBC687 and V1735 Cyg seem to exhibit an asymetric $\mathrm{Pa} \beta$ line profile similar to the one of $\mathrm{Z} \mathrm{CMa}$, although the wavelength sampling of these comparison spectra is lower than SINFONI's. The line profile of the FUOR does not change significantly during the one-year lag corresponding to the OSIRIS and SINFONI spectra. The line properties are clearly not compatible with the hypothesis of an unresolved binary.

The colors of the FUOR recorded in 1990 and during the 2008 outburst (Fig. 16) are redder than those of pre-mainsequence stars earlier than $\mathrm{K} 4(J-H=0.60, H-K=0.17$, Pecaut \& Mamajek 2013) and typical of classical FU Orionis stars (see Fig. 4 of Greene et al. 2008). The variations in position of the source in color-color and color-magnitude diagrams during and after the 2008 outburst is almost perpendicular to the interstellar extinction vector. The colors while the system was returning to quiescence fall outside of the locus of FU Ors and classical T Tauri stars of Meyer et al. (1997). It is difficult to relate this behavior to those of other FU Ors since there has not been an extensive follow-up of the NIR photometry of these objects so far. Our observations are consistent with the variation in optical colors, in the period which show a progressive bluing of $\mathrm{Z} \mathrm{CMa}$ colors consistent with the behavior of the $\mathrm{HBe}$ in the NIR, but also another dependence that could be induced by 
Table 4. Line identification in the $1.1-2.5 \mu \mathrm{m}$ spectrum of the FUOR components.

\begin{tabular}{|c|c|c|}
\hline $\begin{array}{l}\lambda_{\mathrm{obs}} \\
(\mu \mathrm{m})\end{array}$ & Element & Transition \\
\hline 1.10399 & $\mathrm{TiO}$ & $0-0$ band of $\Phi\left(b^{1} \Pi-d^{1} \Sigma\right)$ \\
\hline 1.11752 & $\mathrm{TiO}$ & $1-1$ band of $\Phi\left(b^{1} \Pi-d^{1} \Sigma\right)$ \\
\hline 1.13502 & $\mathrm{TR}$ & $\ldots$ \\
\hline 1.19745 & $\mathrm{TR}$ & $\ldots$ \\
\hline $1.17-1.20$ & VO & $0-1$ band of $A^{4} \Pi-X^{4} \Sigma-$ \\
\hline 1.24339 & $\mathrm{TiO}$ & $0-1$ band of $\Phi\left(b^{1} \Pi-d^{1} \Sigma\right)$ \\
\hline 1.25341 & $\mathrm{TR}$ & $\ldots$ \\
\hline 1.25643 & {$[\mathrm{Fe} \mathrm{II}] ?$} & $a^{6} D_{9 / 2}-a^{4} D_{7 / 2}$ \\
\hline 1.25739 & $\mathrm{TiO}$ & $1-2$ band of $\Phi\left(b^{1} \Pi-d^{1} \Sigma\right)$ \\
\hline 1.27870 & $\mathrm{TR}$ & $\ldots$ \\
\hline 1.28104 & $\mathrm{H} \mathrm{I}(\mathrm{Pa} \beta)$ & $3_{*}-5_{*}$ \\
\hline 1.34830 & $\mathrm{TR}$ & $\ldots$ \\
\hline $1.3-1.55$ & $\mathrm{H}_{2} \mathrm{O}$ & $\begin{array}{l}2 v_{3}, v_{1}+v_{2}, 2 v_{1} \\
2 v_{2}+v_{3}, v_{1}+2 v_{2}\end{array}$ \\
\hline 1.50436 & TR & $\ldots$ \\
\hline 1.50898 & $\mathrm{TR}$ & $\ldots$ \\
\hline $1.570-1.574$ & TR & $\ldots$ \\
\hline $1.597-1.618$ & ${ }^{12} \mathrm{CO} ?$ & 5-2 band of $X^{1} \Sigma^{+}-X^{1} \Sigma^{+}$ \\
\hline $1.619-1.634$ & ${ }^{12} \mathrm{CO} ?$ & 6-3 band of $X^{1} \Sigma^{+}-X^{1} \Sigma^{+}$ \\
\hline $1.721-1.732$ & TR & $\ldots$ \\
\hline $1.70-2.05$ & $\mathrm{H}_{2} \mathrm{O}$ & $v_{2}+v_{3}, v_{1}+v_{2}, 3 v_{2}$ \\
\hline $1.747-1.800$ & $\mathrm{TR}$ & $\ldots$ \\
\hline $2.00-2.03$ & TR & $\ldots$ \\
\hline $2.05-2.08$ & $\mathrm{TR}$ & $\ldots$ \\
\hline 2.10645 & $\mathrm{TR}$ & $\ldots$ \\
\hline 2.20770 & $\mathrm{TR}$ & $\ldots$ \\
\hline 2.29452 & ${ }^{12} \mathrm{CO}$ & 2-0 band of $X^{1} \Sigma^{+}-X^{1} \Sigma^{+}$ \\
\hline 2.32382 & ${ }^{12} \mathrm{CO}$ & $3-1$ band of $X^{1} \Sigma^{+}-X^{1} \Sigma^{+}$ \\
\hline $2.35-2.40$ & TR & $\ldots$ \\
\hline $2.3-2.4$ & $\mathrm{H}_{2} \mathrm{O}$ & $v_{1}, v_{3}, 2 v_{2}$ \\
\hline
\end{tabular}

the variability of the FUOR component (Grankin \& Artemenko 2009).

The intrinsic variation of the FUOR colors could translate into NIR slope variation of our continuum. In that case, the slope of the spectral continuum of the HBe in the $H$-band OSIRIS data could be uncertain because it has been based on the hypothesis of a non-variation of the spectrum of the FUOR (see Sect. 3.2). This does not change our conclusions on the variation of the $\mathrm{HBe}$ continuum slope, which mostly relies on the broadband NIR colors reported in Table 3.

\subsection{Resolved structures}

The spatial sampling of the SINFONI cubes enable us to look for extended structures down to $\sim 0.15^{\prime \prime}$. We detected in Whelan et al. (2010) the FUOR and HBe jets at a position angle of $\sim 235^{\circ}$ and $245^{\circ}$, respectively into $1.257 \mu \mathrm{m}$ and $1.644 \mu \mathrm{m}$ [FeII] lines. In a recent analysis of $\mathrm{H}_{\alpha}$ and [OI] $(655.6 \mathrm{~nm}$ and
$629.5 \mathrm{~nm}$ ) AO-imaging data (Antoniucci et al. 2016), we report the detection of the wiggling of the FUOR jet. We chose here to re-investigate our data to look for structures in other emission lines in the context of the results of Antoniucci et al. (2016).

We report extended emission at 1.200, 1.205, and 1.533, and $2.098 \mu \mathrm{m}$ following the subtraction of the continuum emission (see Appendix B for the details). We classify these emissions into three categories:

- The $1.533 \mu \mathrm{m}$ emission corresponds to the [Fe II] line seen in our spectrum of the system (Table C.1 and Fig. 9). The emission map at more than $3 \sigma$ the noise level is shown in Fig. 17. The dashed ellipse corresponds to the era within which the continuum substraction leaves strong residuals. The emission is associated with the FUOR micro jet (orange dashed line in the figures). It is elongated along a position angle of $\sim 238^{\circ}$. The emission is detected in the same velocity range at 1.257 and $1.644 \mu \mathrm{m}$. The emission has a sinusoid shape with a $\sim 75$ mas semi-period from -400 to $-100 \mathrm{~km} \mathrm{~s}^{-1}$ that is consistent with the wiggling found by Antoniucci et al. (2016).

- The 1.200 and $1.205 \mu \mathrm{m}$ line emissions may be associated with [Fe II] lines at $1.2000278 \mu \mathrm{m}$ and $1.2054490 \mu \mathrm{m}$, respectively. We assume that we correctly identified the lines to map the emission in the velocity space in Fig. 18. These lines trace an elongated emission at a position angle of $\sim 214^{\circ}$ (green dashed lines in Figs. 17 to 18). The emission is seen from -600 to $110 \mathrm{~km} \mathrm{~s}^{-1}$. Its elongation is in the same direction as the $K 1$ knot reported by Whelan et al. (2010). The structure is not seen in the polarized band imaging data of Canovas et al. (2015) and Liu et al. (2016), but the position angle corresponds to the one of the elongated stream (see Sect. 4.2). The stream does not extend down to $0.15^{\prime \prime}$ however, and does not point toward the HBe (Canovas et al. 2015). Additional observations carried out at these wavelengths with high-order adaptive optic instruments such as VLT/SPHERE (N_Cnt] filter) may help to clarify the nature of this structure.

- A clump is detected at $2.098 \mu \mathrm{m}$ (Fig. 18) at a PA of $164 \pm 3^{\circ}$ and separation of $313 \pm 13$ mas (291-360 au). This clump is at the same position as the polarized clump or arm detected in the $H(1.66 \mu \mathrm{m})$ and $K \mathrm{~s}(2.18 \mu \mathrm{m})$ band by Canovas et al. (2015) and Liu et al. (2016). The He I and $\mathrm{H}_{2}$ emission lines seen in jets and embedded objects in this wavelength range translate into an absolute velocity $\gg 1000 \mathrm{~km} \mathrm{~s}^{-1}$. Plausible explanations are that the line is incorrectly identified, or that the structure is unrelated to the system. We detect another clump at a position angle of $\sim 95^{\circ}$ (labeled as ? in Fig. 18). This additional clump is also found at $2.11 \mu \mathrm{m}$, and could correspond to an emission in the He I line $(2.112583 \mu \mathrm{m})$ blueshifted by -400 to $-200 \mathrm{~km} \mathrm{~s}^{-1}$. Nonetheless, it falls close to the PSF Airy ring of the FUOR which moves with wavelength and might produce some residual emission at the continuum subtraction step.

We were unable to detect any extended structures apart from the probable diffraction spikes associated with the HBe and FUOR point sources in images derived from the cubes collapsed in wavelengths and with the stellar halo of each component removed with a Gaussian smoothing (with a $F W H M=5,10$, and 15 pixels).

\footnotetext{
7 This value is consistent with the interval given in Poetzel et al. (1989) for the large-scale jet and by Whelan et al. (2010) and Antoniucci et al. (2016) for the FUOR micro jet.
} 

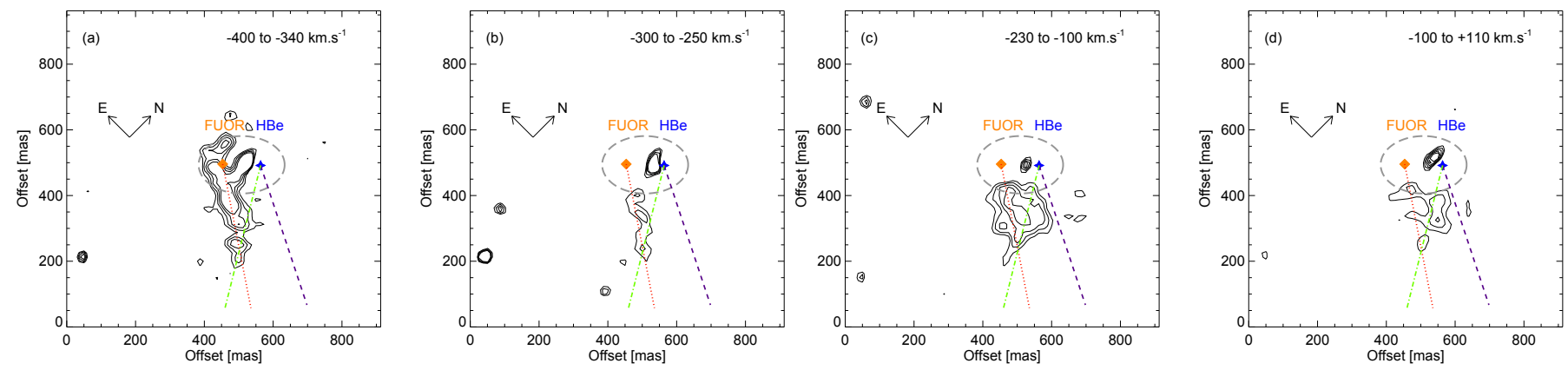

Fig. 17. Extended structures observed in the [Fe II] line at $1.53 \mu \mathrm{m}$.
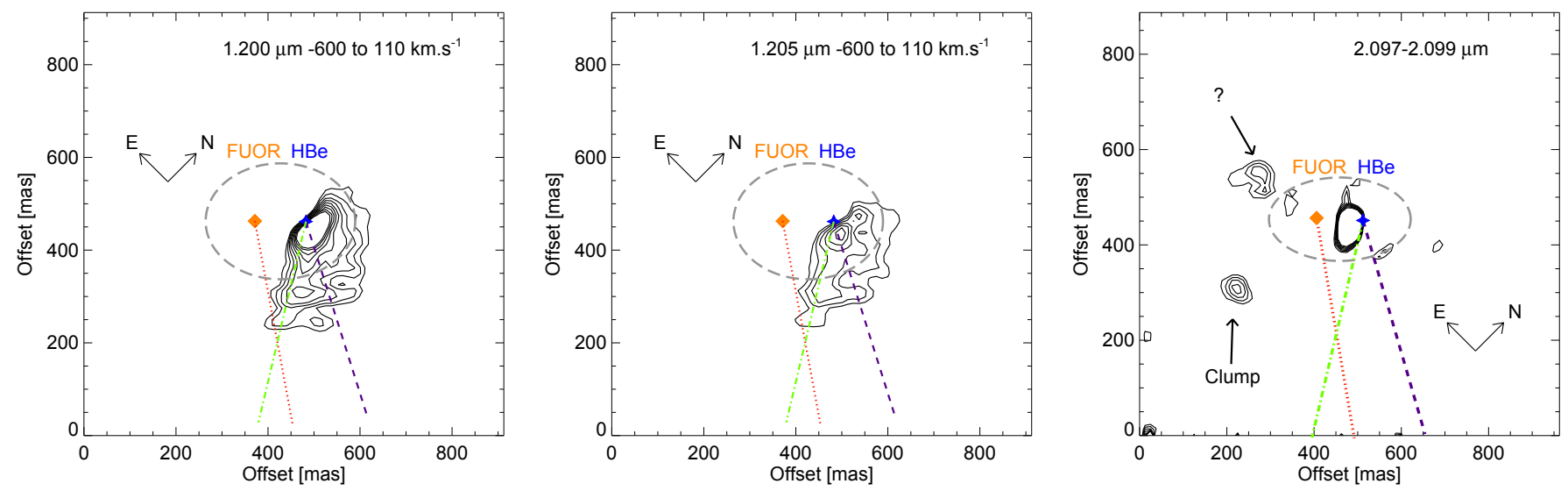

Fig. 18. Extended structures observed at 1.200, 1.205, and $2.098 \mu \mathrm{m}$.

\section{Origins of the outburst: extinction or accretion?}

The architecture of the Z CMa system is reminiscent of those of other EX Ors objects with binary companions such as V1118 Ori (Reipurth et al. 2007), VY Tau (Leinert et al. 1993), and XZ Tau (Hartigan \& Kenyon 2003), altough Z CMa may be the most massive system of this class of outbursting binaries, and the only one with a FU Or companion. These three systems have projected separations of $72 \mathrm{au}, 95 \mathrm{au}$, and 43 au respectively. It is possible that the outbursts of $\mathrm{Z} \mathrm{CMa}$ are related to instabilities in the disk surrounding the $\mathrm{HBe}$ at the corotation radius (e.g., much closer to the star) where gas accumulates until it is accreted during outbursts (D'Angelo \& Spruit 2012). This scenario may explain the behavior of EX Lup (D'Angelo \& Spruit 2012; Banzatti et al. 2015). In this case, are our spectrophotometric data of the $\mathrm{HBe}$ of $\mathrm{Z} \mathrm{CMa}$ more compatible with the scenario of an accretion+ejection event, or a variation of the extinction in the line of sight?

The fact that the jet of the HBe wiggles (Whelan et al. 2010), the existence of knots along the direction of the HBe outflow, and the change in velocity of the blueside wing of the Paschen $\beta$ line associated with the HBe outflow suggest that the 2008 outburst and spectrophotometric properties of the most massive star of the system are related to an accretion+ejection event. The spectrum of the $\mathrm{HBe}$, quite similar to the one of the prototype EX Lup, strengthens this conclusion. Nonetheless, because (i) the $\mathrm{Br} \gamma$ line emission may originate at least partly in a disk wind (Benisty et al. 2010) and (ii) the empirical relation between the $\mathrm{Br} \gamma$ flux and the accretion rate seems to break down in the Be regime (Donehew \& Brittain 2011), the accretion rate as well as the extinctions from line ratio $(\mathrm{Pa} \beta / \mathrm{Br} \gamma)$ cannot be derived reliably from our NIR spectra.

Szeifert et al. (2010) claimed that the 2008 outburst is related to a change in extinction from the dust cocoon surrounding the HBe based on the level of optical polarization in both continuum and spectral lines along a position angle roughly perpendicular to the outflow launched by the HBe. The characteristics of the HBe component during the 2008 outburst are not fully typical of EX Or objects and outbursting stars. The variation in the NIR continuum slope (colors) of the HBe with respect to the absolute NIR flux is anticorrelated with the one of V1647 Ori, PV Cep, or V1118 Ori (Gibb et al. 2006; Lorenzetti et al. 2009; Giannini et al. 2016a). The emission lines of these four reference objects are correlated with their overall NIR brightness (outburst or quiescence) like $\mathrm{Z} \mathrm{CMa}$, based on the current stage of observations (temporal baseline, frequency of the measurements) of all those systems. Stelzer et al. (2009) noted that V1118 Ori had a different X-ray luminosity and temperature variation during an outburst with respect to the behavior of $\mathrm{Z} \mathrm{CMa}$ during the 2008 outburst, thus pointing to a different outburst mechanism for these systems. Z CMa may resemble the case of PTF 10nvg where the spectrophotometry of the system seems to be driven by the rotating circumstellar disk material located at short separation $(<1 \mathrm{au})$ which causes semi-periodic dimming, and by the emission of excited zones in that same disk (Kóspál et al. 2013). PTF 10nvg reproduces the behavior of the absolute flux, NIR spectral slope, and NIR emission lines of Z CMa.

The 2008 outburst can be placed in context now that the system has been monitored for several years before and after the outburst (Fig. 1). VDA04 noted that photometric variations of 
ZCMa between 1987 February and 1991 closely follow a behavior of redder optical colors when the system becomes fainter. It is therefore quite possible that the 2008 and 1987 outbursts of $\mathrm{Z}$ CMa have close origins. At least, both outburst were caused by the HBe. We also note that the 2008 outburst was followed closely by another one in 2011 with similar $V$-band brightness. We can speculate that the 2008 and 2011 outbursts might have been caused by one single accretion+ejection event modulated by absorption in the line of sight during the quiescent stage of 2010 (OSIRIS spectra). This would explain why the CO bandhead is seen in emission during the 2006 quiescent stage (the last one before the 2008 outburst) and then could not be seen at all in the OSIRIS spectrum taken during the 2009 quiescent period (veiling).

\section{Conclusion}

We have obtained multi-epoch NIR astrometric, photometric, and spectroscopic data of the young system Z CMa using adaptive-optics-fed instruments. Our data enabled us to obtain resolved $1-4 \mu \mathrm{m}$ photometry as well as medium-resolution $(R \sim$ 2000-4000) spectra of each component.

Our photometry confirms that the Herbig component of the system is the source driving the ourburst. Its spectrum in the outburst stage is characteristic of EX Ors such as EX Lupi and of embedded young stellar objects. The emission lines, NIR luminosity, spectral continuum (colors) of the Herbig component of ZCMa, and their evolution with time, suggest that the properties of the object are driven both by accretion+ejection changes and variable extinction in the line of sight. We find in particular a correlation between the strength of the $\mathrm{Br} \gamma$ emission and the brightness of the system. Nevertheless, we cannot have a direct access to the reddenning and accretion rate because the emission lines that would enable for an estimation originate in different locations around the Herbig objet (disk, outflow). This prevents us from firmly linking the spectrophotometric properties of the system to their origins. The FU Or companion also experienced color variations at a constant luminosity during the outburst that do not seem to be related to variable extinction.

We detected the extended emission the FUOR jet at $1.53 \mu \mathrm{m}$ ([Fe II] line). We also resolved an extended emission at 1.200 and $1.205 \mu \mathrm{m}$ that extends along a position angle of $214^{\circ}$, and whose origin is unclear. To conclude, we identified a point source at $2.098 \mu \mathrm{m}$ that is concomitant with a polarized clump seen in multiple NIR imaging data.

The system is undergoing a new outburst in 2016. Similar observations as conducted in our study, but with a better temporal sampling, coupled to observations at longer wavelengths with the mid-infrared instrument VISIR (Lagage et al. 2004) and ALMA may dramatically improve our understanding of the architecture of the system, and how it relates to the accretion+ejection diagnostics used in the NIR.

Acknowledgements. We would like to thank particularly the staff of ESOVLT for their support at the telescope as well as Sasha Hinkley and Mario Van Den Acker, who kindly provided their NIR photometry and spectra of the Z CMa system. We are very grateful to Mike Connelley, Kevin Covey, Lynne Hillenbrand, Erika Gibb, and Alessio Caratti o Garatti for providing their spectra of outbursting and embedded objects. We acknowledge with thanks the variable star observations from the AAVSO International Database contributed by observers worldwide (Eric Blown in particular) and used in this research. This work was supported by the Momentum grant of the MTA CSFK Lendület Disk Research Group. We acknowledge partial financial support from the Programmes Nationaux de Planétologie et de Physique Stellaire (PNP \& PNPS) and the Agence Nationale de la Recherche, in France.

\section{References}

Abuter, R., Schreiber, J., Eisenhauer, F., et al. 2006, New Astron. Rev., 50, 398 Alonso-Albi, T., Fuente, A., Bachiller, R., et al. 2009, A\&A, 497, 117 Antoniucci, S., Nisini, B., Giannini, T., \& Lorenzetti, D. 2008, A\&A, 479, 503 Antoniucci, S., Arkharov, A. A., Giannini, T., et al. 2009, ATel, 2024, 1 Antoniucci, S., García López, R., Nisini, B., et al. 2011, A\&A, 534, A32 Antoniucci, S., Podio, L., Nisini, B., et al. 2016, A\&A, 593, L13

Banzatti, A., Pontoppidan, K. M., Bruderer, S., Muzerolle, J., \& Meyer, M. R. 2015, ApJ, 798, L16

Barth, W., Weigelt, G., \& Zinnecker, H. 1994, A\&A, 291, 500

Benisty, M., Malbet, F., Dougados, C., et al. 2010, A\&A, 517, L3

Bergfors, C., Brandner, W., Bonnefoy, M., et al. 2016, MNRAS, 456, 2576

Bonnefoy, M., Chauvin, G., Dumas, C., et al. 2009, A\&A, 506, 799

Bonnefoy, M., Chauvin, G., Lagrange, A.-M., et al. 2014, A\&A, 562, A127

Bonnet, H., Ströbele, S., Biancat-Marchet, F., et al. 2003, in SPIE Conf. Ser. 4839, eds. P. L. Wizinowich, \& D. Bonaccini, 329

Bonnet, H., Abuter, R., Baker, A., et al. 2004, The Messenger, 117, 17

Canovas, H., Min, M., Jeffers, S. V., Rodenhuis, M., \& Keller, C. U. 2012, A\&A, 543, A70

Canovas, H., Perez, S., Dougados, C., et al. 2015, A\&A, 578, L1

Caratti o Garatti, A., Garcia Lopez, R., Weigelt, G., et al. 2013, A\&A, 554, A66

Clariá, J. J. 1974, A\&A, 37, 229

Connelley, M. S., \& Greene, T. P. 2010, AJ, 140, 1214

Cooper, H. D. B., Lumsden, S. L., Oudmaijer, R. D., et al. 2013, MNRAS, 430, 1125

Covey, K. R., Hillenbrand, L. A., Miller, A. A., et al. 2011, AJ, 141, 40

Covino, E., Terranegra, L., Vittone, A. A., \& Russo, G. 1984, AJ, 89, 1868

D'Angelo, C. R., \& Spruit, H. C. 2012, MNRAS, 420, 416

Devillard, N. 1997, The Messenger, 87, 19

Donehew, B., \& Brittain, S. 2011, AJ, 141, 46

Dumas, C., Terrile, R. J., Brown, R. H., Schneider, G., \& Smith, B. A. 2001, AJ, 121,1163

Eisenhauer, F., Abuter, R., Bickert, K., et al. 2003a, in SPIE Conf. Ser. 4841, eds. M. Iye, \& A. F. M. Moorwood, 1548

Eisenhauer, F., Tecza, M., Thatte, N., et al. 2003b, The Messenger, 113, 17

Eisner, J. A., Hillenbrand, L. A., \& Stone, J. M. 2014, MNRAS, 443, 1916

Garcia, P. J. V., Thiébaut, E., \& Bacon, R. 1999, A\&A, 346, 892

Ghez, A. M., Salim, S., Weinberg, N. N., et al. 2008, ApJ, 689, 1044

Giannini, T., Lorenzetti, D., Antoniucci, S., et al. 2016a, ApJ, 819, L5

Giannini, T., Lorenzetti, D., Harutyunyan, A., et al. 2016b, A\&A, 588, A20

Gibb, E. L., Rettig, T. W., Brittain, S. D., et al. 2006, ApJ, 641, 383

Grankin, K. N., \& Artemenko, S. A. 2009, Information Bulletin on Variable Stars, 5905, 1

Greene, T. P., \& Lada, C. J. 1996, AJ, 112, 2184

Greene, T. P., Aspin, C., \& Reipurth, B. 2008, AJ, 135, 1421

Haas, M., Christou, J. C., Zinnecker, H., Ridgway, S. T., \& Leinert, C. 1993, A\&A, 269, 282

Hartigan, P., \& Kenyon, S. J. 2003, ApJ, 583, 334

Hartmann, L., \& Kenyon, S. J. 1985, ApJ, 299, 462

Hartmann, L., \& Kenyon, S. J. 1996, ARA\&A, 34, 207

Hartmann, L., Kenyon, S. J., Hewett, R., et al. 1989, ApJ, 338, 1001

Hessman, F. V., Eisloeffel, J., Mundt, R., et al. 1991, ApJ, 370, 384

Hillenbrand, L. A., Miller, A. A., Covey, K. R., et al. 2013, AJ, 145, 59

Hinkley, S., Hillenbrand, L., Oppenheimer, B. R., et al. 2013, ApJ, 763, L9

Hummer, D. G., \& Storey, P. J. 1987, MNRAS, 224, 801

Humphreys, R. M. 1978, ApJS, 38, 309

Kaltcheva, N. T., \& Hilditch, R. W. 2000, MNRAS, 312, 753

Kelly, D. M., Rieke, G. H., \& Campbell, B. 1994, ApJ, 425, 231

Koresko, C. D., Beckwith, S. V. W., Ghez, A. M., Matthews, K., \& Neugebauer,

G. 1991, AJ, 102, 2073

Kóspál, Á., Ábrahám, P., Acosta-Pulido, J. A., et al. 2011a, A\&A, 527, A133

Kóspál, Á., Ábrahám, P., Goto, M., et al. 2011b, ApJ, 736, 72

Kóspál, Á., Ábrahám, P., Acosta-Pulido, J. A., et al. 2013, A\&A, 551, A62

Kraus, M., Krügel, E., Thum, C., \& Geballe, T. R. 2000, A\&A, 362, 158

Lagage, P. O., Pel, J. W., Authier, M., et al. 2004, The Messenger, 117, 12

Leinert, C., Zinnecker, H., Weitzel, N., et al. 1993, A\&A, 278, 129

Lenzen, R., Hartung, M., Brandner, W., et al. 2003, in SPIE Conf. Ser. 4841, eds.

M. Iye, \& A. F. M. Moorwood, 944

Li Causi, G., Antoniucci, S., \& Tatulli, E. 2008, A\&A, 479, 589

Liljeström, T., \& Olofsson, G. 1997, ApJ, 478, 381

Liu, H. B., Takami, M., Kudo, T., et al. 2016, Sci. Adv., 2, 1500875

Lorenzetti, D., Giannini, T., Calzoletti, L., et al. 2006, A\&A, 453, 579

Lorenzetti, D., Larionov, V. M., Giannini, T., et al. 2009, ApJ, 693, 1056

Lorenzetti, D., Antoniucci, S., Giannini, T., et al. 2012, ApJ, 749, 188

Malbet, F., Rigaut, F., Bertout, C., \& Lena, P. 1993, A\&A, 271, L9

Martin, S. C. 1997, ApJ, 478, L33 
Meyer, M. R., Calvet, N., \& Hillenbrand, L. A. 1997, AJ, 114, 288

Millan-Gabet, R., \& Monnier, J. D. 2002, ApJ, 580, L167

Millan-Gabet, R., Schloerb, F. P., \& Traub, W. A. 2001, ApJ, 546, 358

Millan-Gabet, R., Monnier, J. D., Akeson, R. L., et al. 2006, ApJ, 641, 547

Monnier, J. D., Millan-Gabet, R., Billmeier, R., et al. 2005, ApJ, 624, 832

Murakawa, K., Lumsden, S. L., Oudmaijer, R. D., et al. 2013, MNRAS, 436, 511

Muzerolle, J., Hartmann, L., \& Calvet, N. 1998, AJ, 116, 2965

Nisini, B., Antoniucci, S., Giannini, T., \& Lorenzetti, D. 2005, A\&A, 429. 543

Pecaut, M. J., \& Mamajek, E. E. 2013, ApJS, 208, 9

Poetzel, R., Mundt, R., \& Ray, T. P. 1989, A\&A, 224, L13

Rayner, J. T., Cushing, M. C., \& Vacca, W. D. 2009, ApJS, 185, 289

Reipurth, B., \& Aspin, C. 1997, AJ, 114, 2700

Reipurth, B., Aspin, C., Beck, T., et al. 2007, AJ, 133, 1000
Rousset, G., Lacombe, F., Puget, P., et al. 2003, in SPIE Conf. Ser. 4839, eds. P. L. Wizinowich, \& D. Bonaccini, 140

Sembach, K. R., \& Savage, B. D. 1992, ApJS, 83, 147

Stelzer, B., Hubrig, S., Orlando, S., et al. 2009, A\&A, 499, 529

Szeifert, T., Hubrig, S., Schöller, M., et al. 2010, A\&A, 509, L7

Thiebaut, E., Bouvier, J., Blazit, A., et al. 1995, A\&A, 303, 795

van Dam, M. A., Le Mignant, D., \& Macintosh, B. A. 2004, Appl. Opt., 43, 5458

van den Ancker, M. E., Blondel, P. F. C., Tjin A Djie, H. R. E., et al. 2004, MNRAS, 349, 1516

Veran, J.-P., \& Rigaut, F. J. 1998, in SPIE 3353, eds. D. Bonaccini, \& R. K. Tyson, 426

Whelan, E. T., Dougados, C., Perrin, M. D., et al. 2010, ApJ, 720, L119

Whitney, B. A., Clayton, G. C., Schulte-Ladbeck, R. E., et al. 1993, ApJ, 417, 687 


\section{Appendix A: Details on modeling the $\mathrm{CO}$ emission}

We first considered a disk defined by inner and outer radii $\left(R_{\mathrm{in}}\right.$, $\left.R_{\text {out }}\right)$ and an inclination $(i)$. The radial velocity was computed for each point of the disk assuming that the disk material is in Keplerian rotation around a star with a mass $M_{\star}$. As for the $\mathrm{CO}$ emission, we used Eq. (10) from Kraus et al. (2000) to calculate the absorption coefficient $\kappa$. A simple Gaussian was assumed for the spectral function. This accounts for the thermal and turbulent broadening and for a medium-resolution spectrum. $K$ was multipled with the column density of CO to obtain the optical depth $\tau$. We assumed a simple slab, so that the column density and excitation temperature of $\mathrm{CO}\left(T_{\mathrm{ex}}\right)$ were identical at every location in the disk. We calculated the intensity $I$ following:

$I=B B \times\left(1-\mathrm{e}^{(-\tau / \cos (i))}\right)$

where $B B$ is the Planck function corresponding to the excitation temperature.

The computed spectrum was redshifted (or blueshifted) by the radial velocity appropriate for each point of the disk. We finally integrated over the whole disk in each spectral channel and smoothed the resulting spectrum to account for the finite instrumental spectral resolution of SINFONI.
We varied $T_{\text {ext }}$ between $1500 \mathrm{~K}$ and $4500 \mathrm{~K}$, in steps of $150 \mathrm{~K}$, and a column density $N_{\mathrm{CO}}$ between $10^{17.6} \mathrm{~cm}^{-2}$ and $10^{19.8} \mathrm{~cm}^{-2}$ in logarithmic scale, in steps of 0.1 in the exponent. We took $R_{\text {in }}=0$ au, $i=20^{\circ}$, and $M_{\star}=16 M_{\odot}$. We considered $R_{\text {out }}$ from 0.5 to $3.5 \mathrm{au}$, with 0.5 au increments.

We compared the first two overtones of the HBe (following the removal of the continuum in the SINFONI spectrum) to the grid of 3381 model spectra using a $\chi_{2}$ minimization.

\section{Appendix B: Removal of the continuum emission}

The removal of the spectral continuum in the SINFONI datacubes is required to look for faint structures close to the binary. This is usually done by fitting spaxel to spaxel the spectral continuum around the object line. Here, the intrinsically different continua from the FUOR and $\mathrm{HBe}$ made this removal more complicated. We found that using neighboring cube slices as reference PSF provided a good solution to remove most of the continuum flux of each component. The technique works better than the subtraction in the spectral range (fitting a low-order polynomial) or the spatial subtraction of a PSF model of each component using the extracted cubes derived from the CLEAN 3D algorithm (see Sect. 3.2). 


\section{Appendix C: Additional tables}

Table C.1. Line identification in the 1.1-2.5 $\mu$ m spectrum of the HBe components.

\begin{tabular}{|c|c|c|c|c|c|c|c|}
\hline $\begin{array}{l}\lambda_{\mathrm{obs}} \\
(\mu \mathrm{m})\end{array}$ & Element & Transition & $\begin{array}{l}\Delta V \\
\left(\mathrm{~km} \mathrm{~s}^{-1}\right)\end{array}$ & $\begin{array}{l}F W H M \\
(\AA)\end{array}$ & $\begin{array}{l}\text { Flux } \\
\left(10^{-12} \mathrm{erg} \mathrm{s}^{-1} \mathrm{~cm}^{-2}\right)\end{array}$ & $\begin{array}{l}E W \\
(\AA)\end{array}$ & Remarks \\
\hline 1.11306 & $\mathrm{Fe}$ II & $4 p^{4} F_{3 / 2}^{\circ}-4 s^{2}{ }^{4} G_{5 / 2}$ & 42 & 13.6 & $1.32 \pm 0.32$ & $-1.48 \pm 0.12$ & $\mathrm{CP}$, Blend with $\mathrm{Fe} \mathrm{I}$ \\
\hline 1.12920 & O I & $3 \mathrm{p}^{3} \mathrm{P}_{2}-3 \mathrm{~d}^{3} \mathrm{D}_{3}^{\circ}$ & 42 & 10.8 & $4.60 \pm 0.39$ & $-5.12 \pm 0.16$ & $\mathrm{CP}$ \\
\hline 1.13370 & C I? & $3 \mathrm{p}^{1} \mathrm{P}_{1}-3 \mathrm{~d}^{1} \mathrm{D}_{2}^{\circ}$ & 71 & $\ldots$ & $\ldots$ & $\ldots$ & $\mathrm{SB}$, or TR? \\
\hline 1.13502 & $\mathrm{TR}$ & $\ldots$ & $\ldots$ & $\ldots$ & $\ldots$ & $\ldots$ & \\
\hline 1.13861 & $\mathrm{Na} \mathrm{I}$ & $3 \mathrm{p}^{2} \mathrm{P}_{1 / 2}^{\circ}-4 \mathrm{~s}^{2} \mathrm{~S}_{1 / 2}$ & 28 & 8.2 & $0.49 \pm 0.21$ & $-0.53 \pm 0.09$ & IP \\
\hline 1.14100 & $\mathrm{Na} \mathrm{I}$ & $3 \mathrm{p}^{2} \mathrm{P}_{3 / 2}^{\circ}-4 s^{2} \mathrm{~S}_{1 / 2}$ & 68 & 7.2 & $0.65 \pm 0.19$ & $-0.72 \pm 0.12$ & \\
\hline 1.14451 & $\mathrm{Fe} \mathrm{I}$ & $\mathrm{b}^{3} \mathrm{P}_{1}-\mathrm{z}^{3} \mathrm{D}_{2}^{\circ}$ & 62 & $\ldots$ & $\ldots$ & $\ldots$ & $\mathrm{SB}, \mathrm{IP}$ \\
\hline 1.15996 & $\mathrm{Fe} \mathrm{I}$ & $a^{5} P_{1}-z^{5} D_{0}^{\circ}$ & 63 & 8.9 & $0.63 \pm 0.07$ & $-0.73 \pm 0.04$ & FB \\
\hline 1.16138 & $\mathrm{Fe} \mathrm{I}$ & $a^{5} P_{2}-z^{5} D_{2}^{\circ}$ & 68 & 7.9 & $1.13 \pm 0.07$ & $-1.29 \pm 0.04$ & FB \\
\hline 1.16348 & C I & $3 \mathrm{p}^{3} \mathrm{D}_{2}-3 \mathrm{~d}^{3} \mathrm{D}_{2}^{\circ}$ & 61 & $\ldots$ & $\ldots$ & $\ldots$ & SB \\
\hline 1.16442 & $\mathrm{Fe} \mathrm{I}$ & $\mathrm{a}^{5} \mathrm{P}_{3}-\mathrm{z}^{5} \mathrm{D}_{3}^{\circ}$ & 59 & 9.3 & $0.99 \pm 0.08$ & $-1.12 \pm 0.04$ & FB \\
\hline 1.16643 & C I & $3 \mathrm{p}^{3} \mathrm{~S}_{1}-3 \mathrm{~d}^{3} \mathrm{P}_{0}^{\circ}$ & 47 & $\ldots$ & $\ldots$ & $\ldots$ & SB \\
\hline 1.16959 & $\mathrm{Fe} \mathrm{I}$ & $a^{5} P_{1}-z^{5} D_{1}^{\circ}$ & 59 & 7.8 & $0.78 \pm 0.07$ & $-0.88 \pm 0.04$ & $\begin{array}{l}\text { Blended with } \\
\text { K I line? }\end{array}$ \\
\hline 1.17586 & C I & $3 \mathrm{p}^{3} \mathrm{D}_{3}-3 \mathrm{~d}^{3} \mathrm{~F}_{4}^{\circ}$ & 41 & 11.2 & $3.23 \pm 0.08$ & $-3.65 \pm 0.03$ & $\begin{array}{l}\text { Blend of } \\
\text { C I transitions }\end{array}$ \\
\hline 1.17891 & $\mathrm{Fe} \mathrm{I}$ & $b^{3} P_{2}-z^{3} D_{3}^{\circ}$ & 54 & 7.8 & $1.37 \pm 0.08$ & $-1.54 \pm 0.03$ & $\ldots$ \\
\hline 1.18340 & $\mathrm{Mg} \mathrm{I}$ & $3 \mathrm{p}^{1} \mathrm{P}_{1}^{\circ}-4 \mathrm{~s}^{l} \mathrm{~S}_{0}$ & 54 & $\ldots$ & $\ldots$ & $\ldots$ & SB \\
\hline 1.18440 & $\mathrm{Ca}$ II & $5 s^{2} S_{1 / 2}^{1}-5 p^{2} P_{3 / 2}^{\circ}$ & 58 & $\ldots$ & $\ldots$ & $\ldots$ & SB \\
\hline 1.18893 & $\mathrm{Fe} \mathrm{I}$ & $a^{5} P_{2}-z^{5} D_{3}^{\circ}$ & 69 & $\ldots$ & $\ldots$ & $\ldots$ & $\begin{array}{l}\text { SB with } \\
\text { Fe I and C I lines? }\end{array}$ \\
\hline 1.19001 & $\mathrm{C} \mathrm{I}$ & $3 p^{3} D_{3}-3 d^{3} F_{4}^{\circ}$ & 88 & $\cdots$ & $\ldots$ & $\ldots$ & $\begin{array}{l}\text { SB, Blend of } \\
\text { C I transitions }\end{array}$ \\
\hline 1.19560 & $\mathrm{Ca}$ II & $5 \mathrm{~s}^{2} \mathrm{~S}_{1 / 2}-5 \mathrm{p}^{2} \mathrm{P}_{1 / 2}^{\circ}$ & 63 & 9.9 & $1.69 \pm 0.08$ & $-1.87 \pm 0.04$ & FB \\
\hline 1.19745 & TR & $\ldots$ & $\ldots$ & $\ldots$ & $\ldots$ & $\ldots$ & $\ldots$ \\
\hline 1.19780 & $\mathrm{Fe} \mathrm{I}$ & $b^{3} \mathrm{P}_{3}-\mathrm{z}^{3} \mathrm{D}_{4}^{\circ}$ & 30 & 8.5 & $\ldots$ & $\ldots$ & $\begin{array}{l}\text { IP, blended } \\
\text { with TR }\end{array}$ \\
\hline 1.19897 & Si I & $4 s^{3} P_{1}^{\circ}-4 p^{3} D_{2}$ & 44 & $\ldots$ & $\ldots$ & $\ldots$ & $\mathrm{SB}, \mathrm{IP}$ \\
\hline 1.19972 & Si I & $4 s^{3} P_{0}^{\circ}-4 p^{3} D_{1}$ & 46 & $\ldots$ & $\ldots$ & $\ldots$ & SB, IP \\
\hline 1.20377 & Si I & $4 s^{3} \mathrm{P}_{2}^{\circ}-4 \mathrm{p}^{3} \mathrm{D}_{3}$ & 61 & 9 & $1.46 \pm 0.12$ & $-1.62 \pm 0.05$ & $\ldots$ \\
\hline 1.20902 & $\mathrm{Mg} \mathrm{I}$ & $3 \mathrm{~d}^{1} \mathrm{D}_{2}-4 \mathrm{f}^{\mathrm{l}} \mathrm{F}_{3}^{\circ}$ & 69 & 9 & $0.75 \pm 0.12$ & $-0.84 \pm 0.05$ & $\ldots$ \\
\hline 1.21105 & $\mathrm{Si} \mathrm{I}$ & $4 s^{3} \mathrm{P}_{1}^{\circ}-4 \mathrm{p}^{3} \mathrm{D}_{1}$ & 80 & 12.2 & $0.78 \pm 0.13$ & $-0.87 \pm 0.05$ & $\ldots$ \\
\hline 1.22768 & Si I & $4 s^{3} P_{2}^{\circ}-4 p^{3} D_{2}$ & 56 & 10.4 & $0.71 \pm 0.12$ & $-0.79 \pm 0.05$ & $\mathrm{CP}$, blended? \\
\hline 1.24399 & UF & $\ldots$ & $\ldots$ & $\ldots$ & $\ldots$ & $\ldots$ & SB \\
\hline 1.24665 & UF & $\ldots$ & $\ldots$ & $\ldots$ & $\ldots$ & $\ldots$ & SB \\
\hline 1.25681 & C I & $3 \mathrm{p}^{3} \mathrm{P}_{1}-3 \mathrm{~d}^{3} \mathrm{P}_{0}^{\circ}$ & 50 & $\ldots$ & $\ldots$ & $\ldots$ & SB \\
\hline 1.25681 & {$[\mathrm{Fe} \mathrm{II}]$} & $a^{6} D_{9 / 2}-a^{4} D_{7 / 2}$ & -62 & $\ldots$ & $\ldots$ & $\ldots$ & CP, SB \\
\hline 1.26200 & C I & $3 p^{3} \mathrm{P}_{2}-3 d^{3} \mathrm{P}_{2}^{\circ}$ & 47 & $\ldots$ & $\ldots$ & $\ldots$ & IP \\
\hline 1.27870 & $\mathrm{TR}$ & $\ldots$ & $\ldots$ & $\ldots$ & $\ldots$ & $\ldots$ & Weak TR \\
\hline 1.28239 & $\mathrm{H} \mathrm{I}(\mathrm{Pa} \beta)$ & $3-5$ & 42 & 8.7 & $28.17 \pm 0.68$ & $-30.53 \pm 0.14$ & $\begin{array}{l}\text { Component } \\
\text { at }-649 \mathrm{~km} \mathrm{~s}^{-1}\end{array}$ \\
\hline 1.29754 & $\mathrm{TR}$ & & $\ldots$ & $\ldots$ & $\ldots$ & $\ldots$ & $\ldots$ \\
\hline 1.31305 & $\mathrm{Al} \mathrm{I}$ & ${ }^{2} \mathrm{~S}_{1 / 2}-{ }^{2} \mathrm{P}_{3 / 2}^{\circ}$ & 67 & 8.2 & $0.45 \pm 0.13$ & $-0.49 \pm 0.05$ & $\ldots$ \\
\hline 1.31583 & $\mathrm{Al} \mathrm{I}$ & ${ }^{2} \mathrm{~S}_{1 / 2}-{ }^{2} \mathrm{P}_{3 / 2}^{\circ}$ & 78 & 6.6 & $0.53 \pm 0.08$ & $-0.58 \pm 0.04$ & $\ldots$ \\
\hline 1.32941 & Fe I? & $b^{3} \mathrm{G}-\mathrm{z}^{3} \mathrm{~F}^{\odot}$ & 47 & 10.8 & $0.66 \pm 0.26$ & $-0.71 \pm 0.10$ & $\ldots$ \\
\hline 1.34830 & $\mathrm{TR}$ & $\ldots$ & $\ldots$ & $\ldots$ & $\ldots$ & $\ldots$ & $\ldots$ \\
\hline
\end{tabular}

Notes. TR: telluric residuals. FB: line foot blended with other lines. SB: line strongly blended. CP: complex profile. IB: identified only in emission in the spectrum not corrected for telluric absorptions. IP: poor accuracy on the estimation of the line position. UF: unidentified feature. ND: not seen directly. 
M. Bonnefoy et al.: Multi-epoch images and spectra of the components of Z CMa in the near-infrared during the 2008 outburst

Table C.1. continued.

\begin{tabular}{|c|c|c|c|c|c|c|c|}
\hline $\begin{array}{l}\lambda_{\mathrm{obs}} \\
(\mu \mathrm{m})\end{array}$ & Element & Transition & $\begin{array}{l}\Delta V \\
\left(\mathrm{~km} \cdot \mathrm{s}^{-1}\right)\end{array}$ & $\begin{array}{l}F W H M \\
(\AA)\end{array}$ & $\begin{array}{l}\text { Flux } \\
\left(10^{-12} \mathrm{erg} \mathrm{s}^{-1} \mathrm{~cm}^{-2}\right)\end{array}$ & $\begin{array}{l}E W \\
(\AA)\end{array}$ & Remarks \\
\hline 1.48863 & Mg I & $3 d^{3} D_{3}-4 f^{3} F_{4}^{\circ}$ & 85 & .. & & & IB, IP \\
\hline 1.50327 & $\mathrm{Mg} \mathrm{I}$ & $4 s^{3} S_{1}-4 p^{3} P_{2}^{\circ}$ & 60 & $\ldots$ & $\ldots$ & $\ldots$ & $\mathrm{SB}$ \\
\hline 1.50484 & $\mathrm{Mg} \mathrm{I}$ & $4 s^{3} \mathrm{~S}_{1}-4 \mathrm{p}^{3} \mathrm{P}_{1}^{\circ}$ & 69 & $\ldots$ & $\ldots$ & $\ldots$ & SB \\
\hline 1.50484 & H I (Br 23) & $4-23$ & $\ldots$ & $\cdots$ & $\cdots$ & $\begin{array}{l}\cdots \\
\cdots\end{array}$ & ND \\
\hline 1.50436 & $\mathrm{TR}$ & $\ldots$ & $\ldots$ & $\ldots$ & $\ldots$ & $\ldots$ & $\ldots$ \\
\hline 1.50898 & H I (Br 22) & $4-22$ & 47 & 9.9 & $1.48 \pm 0.09$ & $-1.45 \pm 0.04$ & Overlaping TR \\
\hline 1.51405 & H I (Br 21) & $4-21$ & 51 & 14.6 & $1.39 \pm 0.13$ & $-1.36 \pm 0.04$ & $\ldots$ \\
\hline 1.51994 & H I (Br 20) & $4-20$ & 55 & 19.4 & $1.71 \pm 0.44$ & $-1.42 \pm 0.09$ & $\ldots$ \\
\hline 1.52684 & H I (Br 19) & $4-19$ & 62 & 17.5 & $1.13 \pm 0.14$ & $-1.11 \pm 0.07$ & FB \\
\hline 1.53034 & $\mathrm{Fe} \mathrm{I}$ & $\mathrm{e}^{7} \mathrm{D}_{5}-\mathrm{n}^{7} \mathrm{D}_{5}^{\circ}$ & 80 & $\ldots$ & $\ldots$ & $\ldots$ & SB \\
\hline 1.53495 & {$[\mathrm{Fe} \mathrm{II}]$} & $a^{4} F_{9 / 2}-a^{4} D_{5 / 2}^{3}$ & $\ldots$ & $\ldots$ & $\ldots$ & $\ldots$ & ND \\
\hline 1.53495 & H I (Br 18) & $4-18$ & 57 & 16.7 & $1.91 \pm 0.20$ & $-1.89 \pm 0.05$ & $\ldots$ \\
\hline 1.54470 & H I (Br 17) & $4-17$ & 64 & 14 & $1.85 \pm 0.19$ & $-1.81 \pm 0.06$ & $\ldots$ \\
\hline 1.55640 & H I (Br 16) & $4-16$ & 52 & 11.5 & $3.23 \pm 0.38$ & $-3.15 \pm 0.10$ & $\ldots$ \\
\hline 1.55825 & ${ }^{12} \mathrm{CO} ?$ & $3-0$ of $X^{1} \Sigma^{+}-X^{1} \Sigma^{+}$ & $\ldots$ & $\ldots$ & $\ldots$ & $\ldots$ & SB \\
\hline 1.57083 & H I (Br 15) & $4-15$ & 52 & 13.6 & $\cdots$ & $\cdots$ & Overlaping TR \\
\hline $1.570-1.574$ & $\mathrm{TR}$ & $\ldots$ & $\ldots$ & $\ldots$ & $\ldots$ & $\ldots$ & $\ldots$ \\
\hline 1.57558 & $\mathrm{Mg} \mathrm{I}$ & $4 \mathrm{p}^{3} \mathrm{P}_{0}^{\circ}-4 \mathrm{~d}^{3} \mathrm{D}_{1}$ & 19 & $\ldots$ & $\ldots$ & $\ldots$ & $\mathrm{SB}, \mathrm{IP}$ \\
\hline 1.57746 & $\mathrm{Mg} \mathrm{I}$ & $4 p^{3} p_{2}^{\circ}-4 d^{3} D_{3}$ & 73 & $\cdots$ & $\cdots$ & $\cdots$ & SB, IP \\
\hline 1.57746 & ${ }^{12} \mathrm{CO} ?$ & $4-1$ of $X^{1} \Sigma^{+}-X^{1} \Sigma^{+}$ & $\ldots$ & $\ldots$ & $\ldots$ & $\ldots$ & SB \\
\hline 1.58927 & H I (Br 14) & $4-14$ & 136 & $\ldots$ & $\ldots$ & $\ldots$ & $\begin{array}{l}\text { Blended with } \\
\text { Si I line \& TR }\end{array}$ \\
\hline 1.59677 & $\mathrm{Si} \mathrm{I}$ & $4 p^{3} D_{3}-5 s^{3} P_{2}^{\circ}$ & 51 & $\ldots$ & $\ldots$ & $\ldots$ & SB \\
\hline 1.59871 & ${ }^{12} \mathrm{CO} ?$ & $5-2$ of $X^{1} \Sigma^{+}-X^{1} \Sigma^{+}$ & $\ldots$ & $\ldots$ & $? ?$ & $? ?$ & $\ldots$ \\
\hline 1.61173 & H I (Br 13) & $4-13$ & 55 & 18.8 & $1.79 \pm 0.10$ & $-1.73 \pm 0.05$ & Close TR? \\
\hline 1.61962 & ${ }^{12} \mathrm{CO} ?$ & $6-3$ of $X^{1} \Sigma^{+}-X^{1} \Sigma^{+}$ & $\ldots$ & $\ldots$ & $0.94 \pm 0.27$ & $-0.91 \pm 0.09$ & $\ldots$ \\
\hline 1.64163 & H I (Br 12) & $4-12$ & 74 & 18.8 & $\ldots$ & $\ldots$ & $\mathrm{CP}, \mathrm{SB}$ \\
\hline 1.64117 & {$[\mathrm{Fe} \mathrm{II}]$} & $a^{4} F_{9 / 2}-a^{4} D_{7 / 2}$ & $\ldots$ & $\ldots$ & $\ldots$ & $\ldots$ & ND \\
\hline 1.68159 & $\mathrm{H} \mathrm{I}(\mathrm{Br} 11)$ & $4-11$ & 75 & 17.8 & $2.62 \pm 0.44$ & $-2.48 \pm 0.10$ & $\ldots$ \\
\hline 1.68813 & UF & $\ldots$ & $\ldots$ & $\ldots$ & $\ldots$ & $\ldots$ & SB \\
\hline 1.68990 & C I & $3 \mathrm{p}^{1} \mathrm{D}_{2}-3 \mathrm{~d}^{1} \mathrm{~F}_{3}^{\circ}$ & 60 & $\ldots$ & $\ldots$ & $\ldots$ & SB \\
\hline 1.70105 & $\mathrm{He} \mathrm{I}$ & $3 \mathrm{p}^{3} \mathrm{P}^{\circ}-4 \mathrm{~d}^{3} \mathrm{D}^{3}$ & 48 & 9.1 & $\ldots$ & $\ldots$ & Overlaping TR \\
\hline 1.71171 & Mg I & $4 \mathrm{~s}^{1} \mathrm{~S}_{0}-4 \mathrm{p}^{1} \mathrm{P}_{1}^{\circ}$ & 54 & 8.8 & $0.95 \pm 0.70$ & $-0.89 \pm 0.17$ & $\ldots$ \\
\hline $1.721-1.732$ & $\mathrm{TR}$ & $\ldots$ & $\ldots$ & $\ldots$ & $\ldots$ & $\ldots$ & $\cdots$ \\
\hline 1.73713 & H I (Br 10) & $4-10$ & 66 & 15.6 & $\ldots$ & $\cdots$ & Close TR \\
\hline $1.747-1.800$ & $\mathrm{TR}$ & $\ldots$ & $\ldots$ & $\ldots$ & $\ldots$ & $\ldots$ & $\ldots$ \\
\hline $2.00-2.03$ & TR & $\ldots$ & $\ldots$ & $\ldots$ & $\ldots$ & $\ldots$ & $\ldots$ \\
\hline $2.05-2.08$ & TR & $\ldots$ & $\ldots$ & $\ldots$ & $\ldots$ & $\ldots$ & $\ldots$ \\
\hline 2.10645 & TR & $\ldots$ & $\ldots$ & $\ldots$ & $\ldots$ & $\ldots$ & $\ldots$ \\
\hline 2.16657 & $\mathrm{H}$ I $(\mathrm{Br} \gamma)$ & $4-7$ & 51 & 17.4 & $2.87 \pm 0.83$ & $-2.33 \pm 0.09$ & $\ldots$ \\
\hline 2.20690 & $\mathrm{Na} \mathrm{I}$ & $4 s^{2} S_{1 / 2}-4 p^{2} P_{3 / 2}^{\circ}$ & 78 & $\ldots$ & $\ldots$ & $\ldots$ & IB, IP \\
\hline 2.20770 & TR & $\ldots$ & $\ldots$ & $\ldots$ & $\ldots$ & $\ldots$ & $\ldots$ \\
\hline 2.20951 & $\mathrm{Na} \mathrm{I}$ & $4 s^{2} S_{1 / 2}-4 p^{2} P_{1 / 2}^{\circ}$ & 63 & $\ldots$ & $\ldots$ & $\ldots$ & IB, IP \\
\hline 2.29512 & ${ }^{12} \mathrm{CO}$ & $2-0$ of $X^{1} \Sigma^{+}-X^{1} \Sigma^{+}$ & $\ldots$ & $\ldots$ & $13.97 \pm 4.40$ & $-10.82 \pm 0.54$ & $\ldots$ \\
\hline 2.32417 & ${ }^{12} \mathrm{CO}$ & $3-1$ of $\mathrm{X}^{1} \Sigma^{+}-\mathrm{X}^{1} \Sigma^{+}$ & $\ldots$ & $\ldots$ & $8.91 \pm 4.37$ & $-6.78 \pm 0.56$ & $\ldots$ \\
\hline 2.35391 & ${ }^{12} \mathrm{CO}$ & $4-2$ of $X^{1} \Sigma^{+}-X^{1} \Sigma^{+}$ & $\ldots$ & $\ldots$ & $8.66 \pm 4.09$ & $-6.55 \pm 0.63$ & $\ldots$ \\
\hline $2.35-2.40$ & $\mathrm{TR}$ & $\ldots$ & $\ldots$ & $\ldots$ & $\ldots$ & $\ldots$ & $\ldots$ \\
\hline 2.38411 & ${ }^{12} \mathrm{CO}$ & $5-3$ of $X^{1} \Sigma^{+}-X^{1} \Sigma^{+}$ & $\ldots$ & $\ldots$ & $\ldots$ & $\ldots$ & Overlaping TR \\
\hline
\end{tabular}


A\&A 597, A91 (2017)

Table C.2. Variability of the equivalent widths of the Paschen $\beta$ and Brackett $\gamma$ lines, and of the $v=2 \longrightarrow 0{ }^{12} \mathrm{CO}$ overtone for the HBe component.

\begin{tabular}{lllll}
\hline \hline $\begin{array}{l}\text { Date } \\
(\mathrm{DD} / \mathrm{MM} / \mathrm{YYYY})\end{array}$ & Instrument & $\begin{array}{l}E W \mathrm{~Pa} \beta \\
(\AA)\end{array}$ & $\begin{array}{l}E W \mathrm{Br} \gamma \\
(\AA)\end{array}$ & $\begin{array}{l}E W^{12} \mathrm{CO} \\
(\AA)\end{array}$ \\
\hline $17 / 12 / 2006$ & NIRSPEC & $\ldots$ & $\ldots$ & $-3.14 \pm 0.17$ \\
$05 / 12 / 2008$ & AMBER & $\ldots$ & $-4.46 \pm 0.10$ & $\ldots$ \\
$15 / 12 / 2008$ & AMBER & $\ldots$ & $-4.59 \pm 0.08$ & $\ldots$ \\
$16 / 12 / 2008$ & AMBER & $\ldots$ & $-4.21 \pm 0.08$ & $\ldots$ \\
$06 / 02 / 2009$ & SINFONI & $-30.53 \pm 0.14$ & $-2.33 \pm 0.09$ & $-5.51 \pm 0.11$ \\
$22 / 12 / 2009$ & OSIRIS & $-14.67 \pm 0.22$ & $-0.46 \pm 0.10$ & $-2.29 \pm 0.10$ \\
$10 / 01 / 2010$ & AMBER & $\ldots$ & $-0.78 \pm 0.08$ & $\ldots$ \\
\hline
\end{tabular}

